\title{
A rivulet of a power-law fluid with constant width draining down a slowly varying substrate
}

\author{
F. H. H. Al Mukahal, ${ }^{1}$ S. K. Wilson ${ }^{2}$ and B. R. Duffy ${ }^{3}$ \\ Department of Mathematics and Statistics, University of Strathclyde, \\ Livingstone Tower, 26 Richmond Street, Glasgow, G1 1 XH, United Kingdom
}

\begin{abstract}
The flow of a slowly varying rivulet of a power-law fluid with prescribed constant width (i.e. with pinned contact lines) but slowly varying contact angle down a slowly varying substrate, specifically the flow in the azimuthal direction around the outside of a large horizontal circular cylinder, is described. The solution for a rivulet of a perfectly wetting fluid (which can never have constant width) is obtained, and it is shown that, despite having the same local behaviour, the global behaviour of a rivulet of a non-perfectly wetting fluid is qualitatively very different from that of a rivulet with prescribed constant contact angle but slowly varying width. Specifically, it is described how the contact lines of a sufficiently narrow rivulet can remain pinned as it drains all the way from the top to the bottom of the cylinder, but how the contact lines of a wider rivulet de-pin at a critical position on the lower half of the cylinder, and how thereafter it drains to the bottom of the cylinder with zero contact angle and slowly varying width. How the shape of the rivulet and the velocity within it depend on the power-law index $N$ is described in detail. In particular, it is shown that whereas neither the shape of the rivulet nor the velocity within it vary monotonically with $N$, its mass always decreases monotonically with $N$. Despite the limitations of the power-law model, the present results provide rare analytical insight into non-Newtonian rivulet flow, and, in particular, are a useful benchmark for the study of rivulet flow of more realistic non-Newtonian fluids.
\end{abstract}

Keywords: rivulet, power-law fluid, pinned and de-pinned contact lines

\section{Introduction}

Rivulets of viscous fluid occur in many practical contexts, including coating processes (see, for example, Fraysse and Homsy [1]), geophysical flows of lava, ice and mud (see, for example, Balmforth,

\footnotetext{
${ }^{1}$ Email: fatemah.al-mukahalestrath.ac.uk

${ }^{2}$ Author for correspondence. Email: s.k.wilson@strath.ac.uk

${ }^{3}$ Email: b.r.duffy@strath.ac.uk
} 
Craster and Sassi [2]), heat exchangers and trickle-bed reactors (see, for example, Nawrocki and Chuang [3]), the production of microbubbles (see, for example, Herrada et al. [4]), and rain-windinduced vibrations of the cables of cable-stayed bridges (see, for example, Robertson et al. [5]).

Despite the fact that in many practical situations non-Newtonian properties of the fluid play an important role, much of the research on rivulet flow has, perhaps understandably, concerned the special case of a Newtonian fluid. (In the absence of a recent review article, see the representative selection of previous work [6]-[28] and the references therein.) Exceptions to this include the work of Rosenblat [29] on rivulet flow of an elastic fluid, and of Balmforth, Craster and Sassi [2] and Wilson, Duffy and Ross [30] on rivulet flow of a viscoplastic fluid, as well as that of Wilson and Burgess [31], Wilson, Duffy and Hunt [32], and Yatim, Duffy and Wilson [33] on non-uniform rivulets of a powerlaw fluid. There has, of course, been considerable work on other closely related free surface flows of non-Newtonian fluids (see, for example, the recent work [34]-[43]).

Recently Al Mukahal, Duffy and Wilson [44] obtained the solution for unidirectional steady gravitydriven flow of a thin rivulet of a power-law fluid with prescribed volume flux down a planar substrate, and then used it to describe the flow of a slowly varying rivulet of a power-law fluid with prescribed constant contact angle but slowly varying width down a slowly varying substrate. In the present work we show how this solution (together with the corresponding solution for a rivulet of a perfectly wetting fluid, which we obtain here for the first time) can also be used to describe the flow of a slowly varying rivulet of a power-law fluid with prescribed constant width (i.e. with pinned contact lines) but slowly varying contact angle down a slowly varying substrate, specifically the flow in the azimuthal direction around the outside of a large horizontal circular cylinder. Pinned contact lines are likely to occur when the substrate is relatively rough, and rivulets with constant width may result from the manner in which the fluid is initially deposited onto the substrate (e.g. rapid pouring onto the substrate) and/or the texture of the substrate (e.g. a smooth stripe on a rough substrate). In particular, we show that the global behaviour of the rivulet is qualitatively very different from that described by Al Mukahal et al. $[44]$.

\section{Rivulet flow down a planar substrate}

In the present work we shall be concerned with flow of an incompressible power-law fluid with velocity $\mathbf{u}$ and pressure $p$ for which the extra stress $\boldsymbol{\sigma}$ is related to the rate of strain $\mathbf{e}=\frac{1}{2}\left(\nabla \mathbf{u}+(\nabla \mathbf{u})^{\mathrm{T}}\right)$ by $\boldsymbol{\sigma}=2 \mu(q) \mathbf{e}$, where $\mu(q)=\mu_{N} q^{N-1}$ is the shear-rate-dependent viscosity, $q=\left(2 \operatorname{tr}\left(\mathbf{e}^{2}\right)\right)^{1 / 2}$ is the shear rate, and the power-law index $N$ and the consistency parameter $\mu_{N}$ are constants. The fluid is shear 
thinning when $0<N<1$ and shear thickening when $N>1$; the special case $N=1$ corresponds to a Newtonian fluid with constant viscosity $\mu_{1}$. Mass conservation and momentum balance for such a fluid take the forms

$$
\nabla \cdot \mathbf{u}=0, \quad \rho \frac{D \mathbf{u}}{D t}=-\nabla p+\rho \mathbf{g}+\mu_{N} \nabla \cdot\left(q^{N-1}\left(\nabla \mathbf{u}+(\nabla \mathbf{u})^{\mathrm{T}}\right)\right),
$$

where $\rho, \mathbf{g}$ and $t$ denote the fluid density, acceleration due to gravity, and time, respectively.

First we consider unidirectional steady gravity-driven flow of a thin uniform rivulet of a power-law fluid with prescribed volume flux down a planar substrate inclined at an angle $\alpha(0 \leq \alpha \leq \pi)$ to the horizontal. Referred to the natural Cartesian coordinates $O x y z$ (with the $x$-axis down the line of greatest slope of the substrate, the $z$ axis normal to the substrate, and the origin on the substrate at the centre of the rivulet) the substrate is at $z=0$. The free surface of the rivulet is denoted by $z=h$, the semi-width of the rivulet by $a$, and the contact angle by $\beta(\geq 0)$.

We non-dimensionalise and scale the variables by writing

$$
\begin{gathered}
y=\ell y^{*}, \quad a=\ell a^{*}, \quad z=\epsilon \ell z^{*}, \quad h=\epsilon \ell h^{*}, \quad \beta=\epsilon \beta^{*}, \\
u=U u^{*}, \quad p=p_{a}+\rho g \epsilon \ell p^{*}, \quad q=\frac{U}{\epsilon \ell} q^{*}, \quad \mu=\bar{\mu} \mu^{*}, \quad Q=\epsilon \ell^{2} U Q^{*},
\end{gathered}
$$

where $\ell=(\gamma / \rho g)^{1 / 2}$ is the capillary length, in which $\gamma$ is the coefficient of surface tension of the fluid, $\epsilon(\ll 1)$ is the transverse aspect ratio, $U=\left(\rho g \epsilon^{N+1} \ell^{N+1} / \mu_{N}\right)^{1 / N}$ is the appropriate velocity scale, $p_{\mathrm{a}}$ is the atmospheric pressure, $\bar{\mu}=\mu_{N}(U / \epsilon \ell)^{N-1}=\left(\mu_{N}(\rho g \epsilon \ell)^{N-1}\right)^{1 / N}$ is the appropriate viscosity scale, and $Q$ is the volume flux of fluid along the rivulet. In general, there is some freedom in the definition of $\epsilon$. For the present problem it is most convenient to define $\epsilon$ in terms of the prescribed value of the flux, $Q=\bar{Q}$, according to $\epsilon=\left(\mu_{N} \bar{Q}^{N} / \rho g \ell^{3 N+1}\right)^{1 /(2 N+1)}$, in which case $U=\left(\rho g \bar{Q}^{N+1} / \mu_{N} \ell^{N+1}\right)^{1 /(2 N+1)}$, corresponding to taking $\bar{Q}^{*}=1$ without loss of generality. In particular, equation (2) then shows that the thickness of the rivulet varies with the volume flux according to simply $h \propto \bar{Q}^{N /(2 N+1)}$. Note that a different choice of $\epsilon$, namely $\epsilon=\beta$, in which case $U=\left(\rho g \beta^{N+1} \ell^{N+1} / \mu_{N}\right)^{1 / N}$, corresponding to taking $\beta^{*}=1$ without loss of generality, was made by Al Mukahal et al. [44]. From now on we use non-dimensional quantities (with the stars omitted, for clarity).

It may be shown straightforwardly that, at leading order in $\epsilon$, the velocity is due to a longitudinal balance of gravity and viscous effects and is of the form $\mathbf{u}=u(y, z) \mathbf{i}$ with

$$
u=\frac{N}{N+1}(\sin \alpha)^{\frac{1}{N}}\left(h^{\frac{N+1}{N}}-(h-z)^{\frac{N+1}{N}}\right)
$$

the pressure $p=p(z)$ is given by

$$
p=\cos \alpha(h-z)-h^{\prime \prime},
$$


and the cross-sectional free surface profile $h=h(y)$ is due to a transverse balance of gravity and surface-tension effects and satisfies the ordinary differential equation

$$
\left(h^{\prime \prime}-\operatorname{sgn}(\cos \alpha) m^{2} h\right)^{\prime}=0
$$

subject to the boundary conditions

$$
h=0 \text { and } h^{\prime}=\mp \beta \text { at } y= \pm a,
$$

where for later convenience we have defined $m=\sqrt{|\cos \alpha|}$ and a prime denotes differentiation with respect to $y$. The volume flux of fluid along the rivulet is given by

$$
Q=\int_{-a}^{a} \int_{0}^{h} u \mathrm{~d} z \mathrm{~d} y=\frac{N}{2 N+1}(\sin \alpha)^{\frac{1}{N}} \int_{-a}^{a} h^{\frac{2 N+1}{N}} \mathrm{~d} y
$$

\section{Rivulet flow down a slowly varying substrate}

Equations (3)-(7) describe the unidirectional flow of a uniform rivulet of a power-law fluid down a planar substrate. However, they also provide the leading order description of the locally unidirectional flow of a slowly varying rivulet down a slowly varying substrate. In particular, they provide the leading order description of the flow in the azimuthal direction of a slowly varying rivulet around the outside of a large horizontal cylinder. It is rivulets of this type that we consider in the remainder of the present work (although other interpretations, such as, for example, flow down a slowly undulating substrate, are also possible). Specifically, we consider the situation in which both the azimuthal aspect ratio, $\ell / R \ll \epsilon$, and the appropriately defined reduced Reynolds number, $\rho \gamma \epsilon^{4} \ell^{2} / \bar{\mu}^{2} R \ll 1$, are sufficiently small, where $R$ is the radius of the cylinder. In particular, both of these conditions are satisfied if the cylinder is sufficiently large.

Figure 1 shows a sketch of a narrow slowly varying rivulet with prescribed flux $Q=\bar{Q}(=1)$ with prescribed constant semi-width $a=\bar{a}(<\pi)$ and slowly varying contact angle $\beta(>0)$ (i.e. pinned contact lines all the way around the cylinder) draining from the top $(\alpha=0)$ to the bottom $(\alpha=\pi)$ of a large horizontal cylinder. However, in their analysis of this situation for a Newtonian fluid, Paterson et al. [25, Sec. 4] showed that a rivulet can have constant width all the way around the cylinder only if the rivulet is sufficiently narrow (specifically only if $\bar{a} \leq \pi$ ), and that for a wider rivulet (specifically for $\bar{a}>\pi$ ) there is a critical value of $\alpha$ on the lower half of the cylinder, denoted by $\alpha_{\text {depin }}\left(\pi / 2<\alpha_{\text {depin }}<\pi\right)$ and given by

$$
\alpha_{\text {depin }}=\cos ^{-1}\left(-\frac{\pi^{2}}{\bar{a}^{2}}\right) \quad \text { for } \quad \bar{a}>\pi
$$




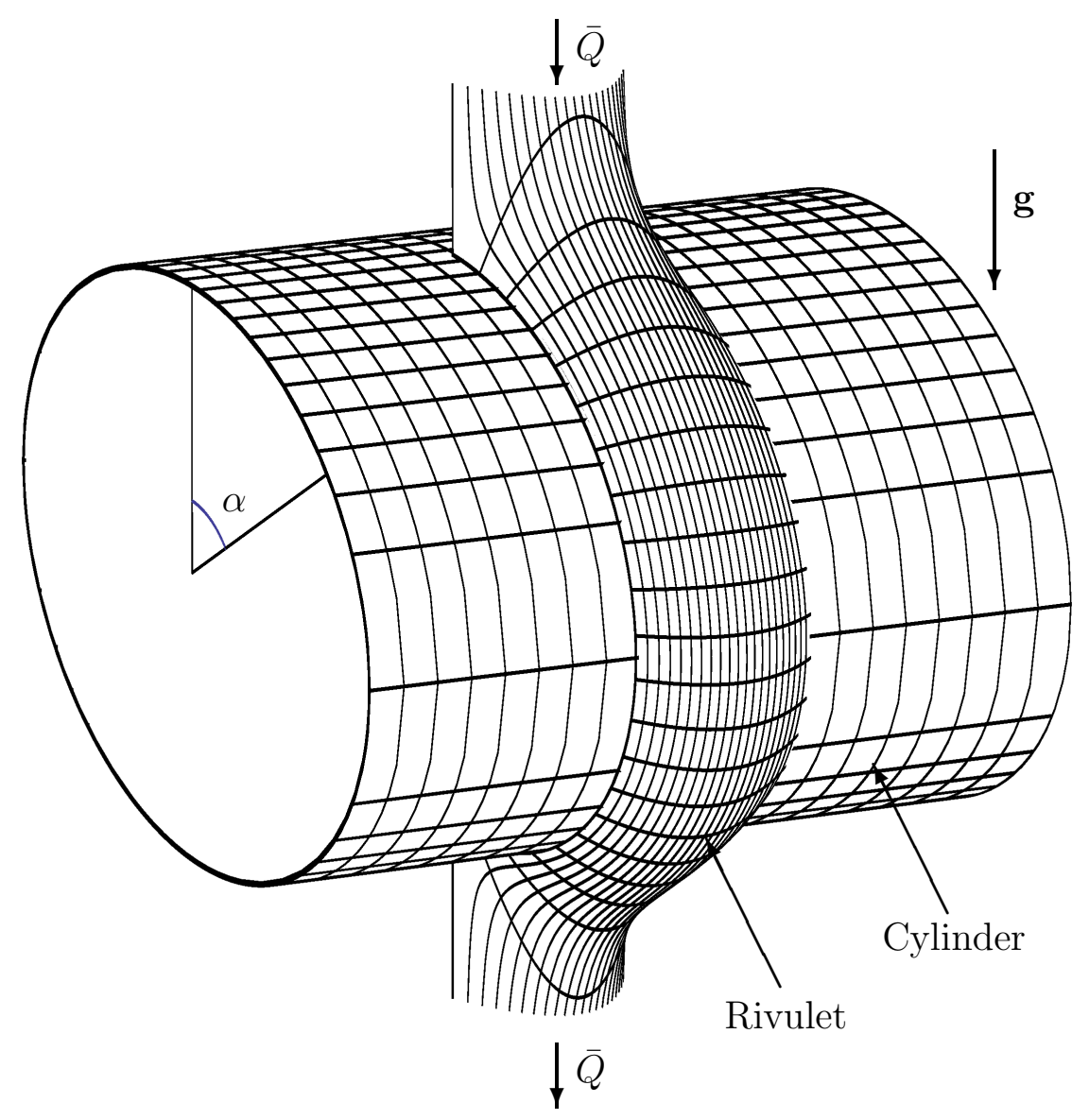

Figure 1: Sketch of a narrow slowly varying rivulet with prescribed flux $Q=\bar{Q}(=1)$ with prescribed constant semiwidth $a=\bar{a}(<\pi)$ and slowly varying contact angle $\beta(>0)$ draining from the top $(\alpha=0)$ to the bottom $(\alpha=\pi)$ of a large horizontal cylinder. 


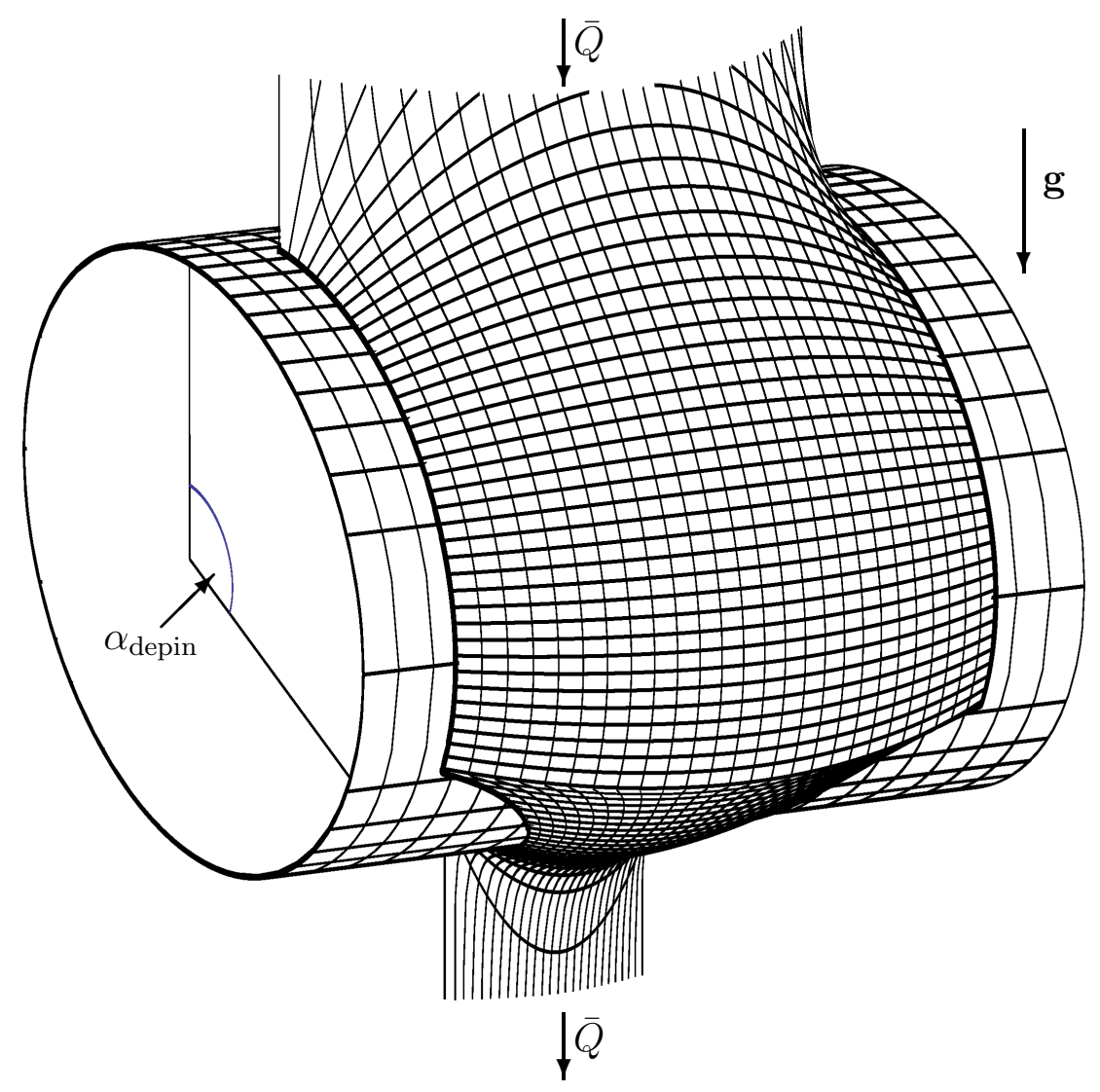

Figure 2: As in Figure 1, except for a wide rivulet with prescribed constant semi-width $a=\bar{a}(>\pi)$ and slowly varying contact angle $\beta$ in $0 \leq \alpha \leq \alpha_{\text {depin }}$, but with zero contact angle $\beta=0$ and slowly varying semi-width $a=\pi / m(\pi \leq a \leq \bar{a})$ in $\alpha_{\text {depin }} \leq \alpha \leq \pi$. 
at which the contact angle is equal to zero, $\beta=0$, and beyond which there is no physically realisable solution with constant semi-width $a=\bar{a}$. [Note that $\alpha_{\text {depin }}$ decreases monotonically with $\bar{a}$, satisfying

$\alpha_{\text {depin }}=\pi+O\left((\bar{a}-\pi)^{1 / 2}\right) \rightarrow \pi^{-}$as $\bar{a} \rightarrow \pi^{+}$and $\alpha_{\text {depin }}=\pi / 2+O\left(\bar{a}^{-2}\right) \rightarrow \pi / 2^{+}$as $\bar{a} \rightarrow \infty$.]

For their problem, Paterson et al. [25] assumed that the contact lines de-pin at $\alpha=\alpha_{\text {depin }}$ and that thereafter the rivulet drains from $\alpha=\alpha_{\text {depin }}$ to the bottom of the cylinder with zero contact angle $\beta=0$ and slowly varying semi-width $a$. [Of course, this behaviour is a special case of the more general scenario in which a rivulet with prescribed constant width de-pins and possibly re-pins at a prescribed non-zero value of the contact angle. This situation was also analysed by Paterson et al. [25, Sec. 6] for a Newtonian fluid, but for brevity is not pursued here.] An important observation is that since (8) does not depend on viscous effects, it also holds for the present case of a power-law fluid (and indeed, for any generalised Newtonian fluid), and hence the same de-pinning of a wide rivulet occurs in the present problem. Figure 2 shows a sketch of a wide rivulet with prescribed constant semi-width $a=\bar{a}(>\pi)$ and slowly varying contact angle $\beta$ in $0 \leq \alpha \leq \alpha_{\text {depin }}$ (i.e. pinned contact lines on the upper part of the cylinder), but with zero contact angle $\beta=0$ and slowly varying semi-width $a=\pi / m$ $(\pi \leq a \leq \bar{a})$ in $\alpha_{\text {depin }} \leq \alpha \leq \pi$ (i.e. de-pinned contact lines on the lower part of the cylinder). Thus in Sec. 4 we obtain the solution for the special case of a rivulet of a perfectly wetting power-law fluid (i.e. a rivulet with zero contact angle), and then in Sec. 5 we use this solution, together with the solution previously obtained by Al Mukahal et al. [44] for the general case of a rivulet of a non-perfectly wetting power-law fluid, to provide the complete description of the rivulets sketched in Figures 1 and 2.

\section{A rivulet of a perfectly wetting fluid $(\beta=0)$}

In the special case of a perfectly wetting fluid $(\beta=0)$ equations $(5)$ and (6) have no solution for $h$ on the upper half of the cylinder (i.e. for $0 \leq \alpha \leq \pi / 2$ ), but on the lower half (i.e. for $\pi / 2<\alpha \leq \pi$ ) they have the simple solution

$$
a=\frac{\pi}{m}, \quad h=\frac{h_{\mathrm{m}}}{2}(1+\cos m y),
$$

where $h_{\mathrm{m}}$ denotes the (as yet unknown) maximum thickness of the rivulet, which occurs at $y=0$. In particular, the solution (9) shows that the semi-width $a$ varies with $\alpha$ (i.e. that a slowly varying rivulet of a perfectly wetting fluid can never have constant width). The maximum velocity, denoted by $u_{\max }$, occurs at $y=0$ and $z=h_{\mathrm{m}}$ and is given by

$$
u_{\max }=\frac{N}{N+1}\left(\sin \alpha h_{\mathrm{m}}^{N+1}\right)^{\frac{1}{N}}
$$




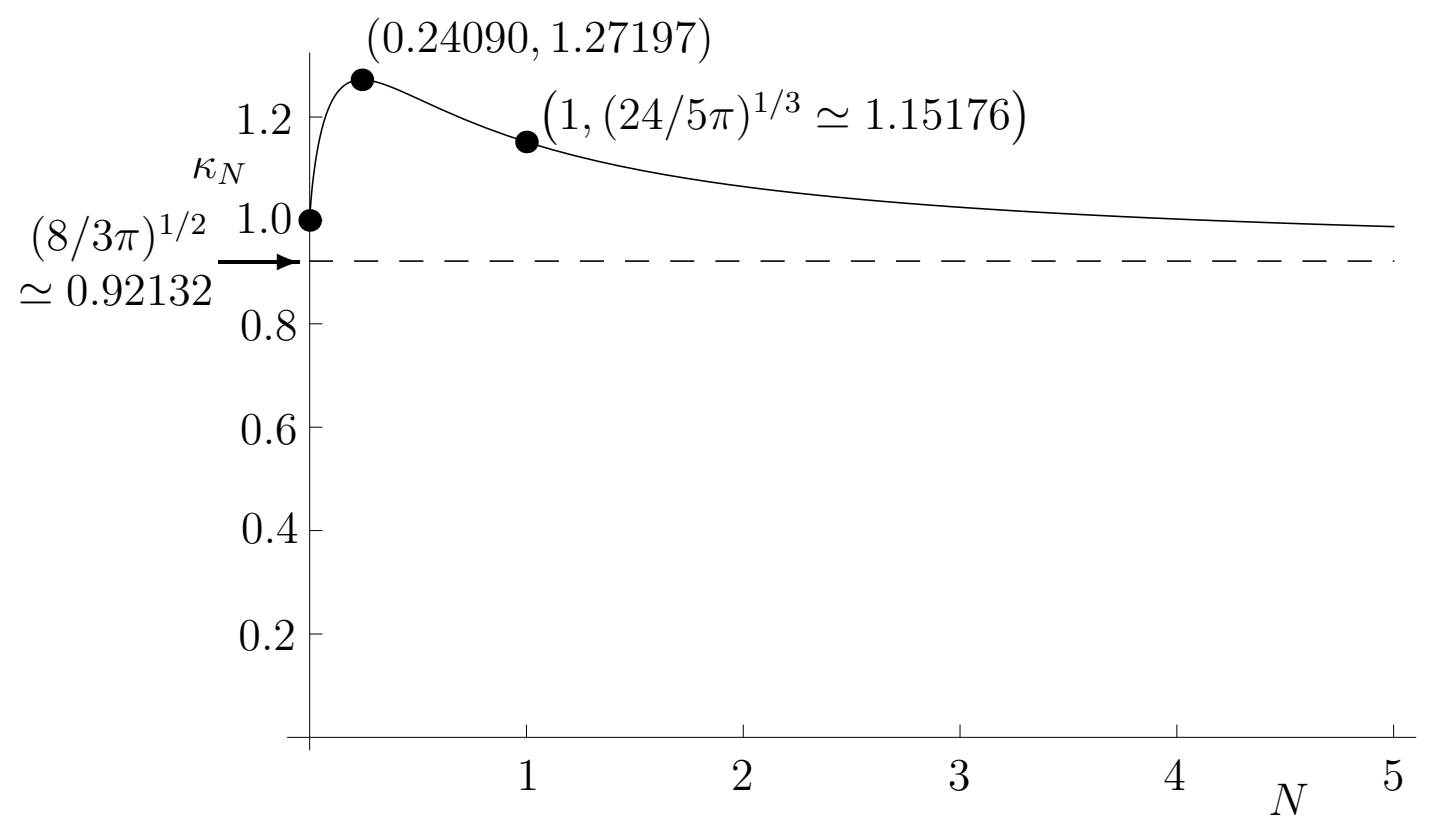

Figure 3: Plot of $\kappa_{N}$ given by (13) as a function of $N$, together with its asymptotic value $(8 / 3 \pi)^{1 / 2} \simeq 0.92132$ in the limit $N \rightarrow \infty$.

To complete the solution we must determine $h_{\mathrm{m}}$ appearing in (9). Performing the quadrature in (7) with $h$ given by (9) we find that the flux $Q$ is given by

$$
Q=\frac{2 \sqrt{\pi} N \Gamma\left(\frac{5 N+2}{2 N}\right)}{(2 N+1) \Gamma\left(\frac{3 N+1}{N}\right)} \frac{(\sin \alpha)^{\frac{1}{N}}}{m} h_{\mathrm{m}}^{\frac{2 N+1}{N}}=\frac{2 N}{2 N+1} \mathrm{~B}\left(\frac{1}{2}, \frac{5 N+2}{2 N}\right) \frac{(\sin \alpha)^{\frac{1}{N}}}{m} h_{\mathrm{m}}^{\frac{2 N+1}{N}},
$$

where $\Gamma$ and $\mathrm{B}$ denote the Gamma and Beta functions, respectively. Prescribing the value of the flux to be $Q=\bar{Q}=1$ yields

$$
h_{\mathrm{m}}=\kappa_{N}\left(\frac{m^{N}}{\sin \alpha}\right)^{\frac{1}{2 N+1}},
$$

where we have introduced $\kappa_{N}$ defined by

$$
\kappa_{N}=\left(\frac{(2 N+1) \Gamma\left(\frac{3 N+1}{N}\right)}{2 \sqrt{\pi} N \Gamma\left(\frac{5 N+2}{2 N}\right)}\right)^{\frac{N}{2 N+1}}=\left[\frac{2 N}{2 N+1} \mathrm{~B}\left(\frac{1}{2}, \frac{5 N+2}{2 N}\right)\right]^{-\frac{N}{2 N+1}} .
$$

Equations (9) and (12) show that, although both $a$ and the form of the cross-sectional free surface profile of the rivulet are independent of $N$, its size (and, in particular, its maximum thickness $h_{\mathrm{m}}$ ) depends on $N$ via the flux. Figure 3 shows a plot of $\kappa_{N}$ given by (13) as a function of $N$, and illustrates that $\kappa_{N}$ satisfies

$$
\kappa_{N}=1-\frac{1}{2} \log \left(4 \pi N^{3}\right) N+O(N \log N)^{2} \rightarrow 1^{+}
$$




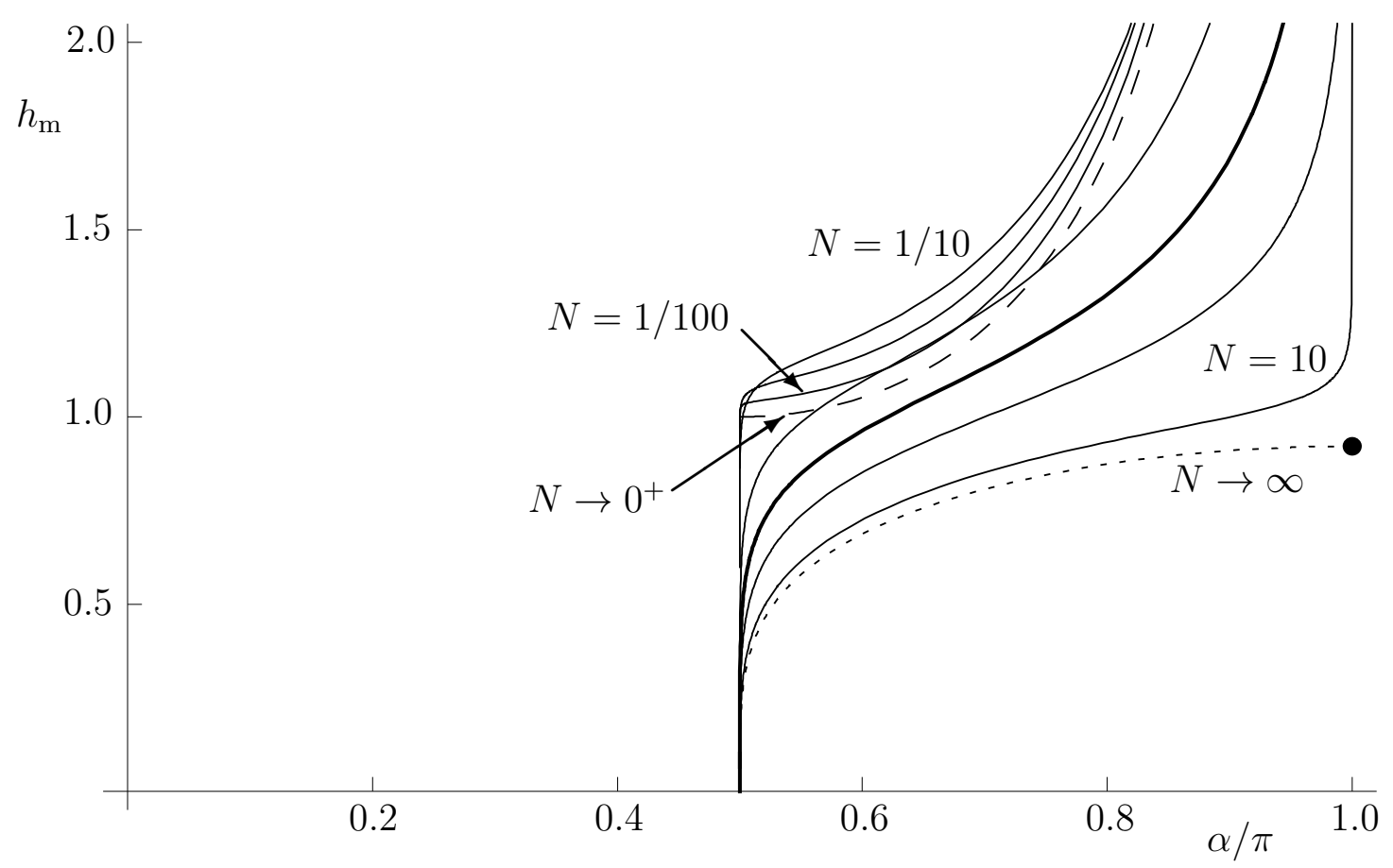

Figure 4: Plots of the maximum thickness $h_{\mathrm{m}}$ of a rivulet of a perfectly wetting fluid given by (12) as a function of $\alpha / \pi$ for $N=1 / 100,1 / 30,1 / 10,1 / 2,1$ (thicker curve), 2 and 10, together with the asymptotic solutions $h_{\mathrm{m}} \sim 1 / \sin \alpha$ in the limit $N \rightarrow 0^{+}$(dashed curve), and $h_{\mathrm{m}} \sim(8 / 3 \pi)^{1 / 2}(-\cos \alpha)^{1 / 4}$ in the limit $N \rightarrow \infty$ (dotted curve).

as $N \rightarrow 0^{+}$, increases to a maximum 1.27197 at $N \simeq 0.24090$, takes the value $(24 / 5 \pi)^{1 / 3} \simeq 1.15176$ at $N=1$, and thereafter decreases monotonically, satisfying

$$
\kappa_{N}=\left(\frac{8}{3 \pi}\right)^{1 / 2}\left[1+\frac{3 \ln 6 \pi-4}{12 N}+O\left(\frac{1}{N^{2}}\right)\right] \rightarrow\left(\frac{8}{3 \pi}\right)^{1 / 2+} \simeq 0.92132^{+}
$$

as $N \rightarrow \infty$. In particular, when $N=1$ equation (12) reduces to the solution in the special case of a Newtonian fluid given by Duffy and Wilson [11], namely $h_{\mathrm{m}}=(24 m /(5 \pi \sin \alpha))^{1 / 3}$.

Figure 4 shows plots of $h_{\mathrm{m}}$ given by (12) as a function of $\alpha / \pi$ for a range of values of $N$, illustrating that in all cases $h_{\mathrm{m}}$ increases monotonically with $\alpha$, and that the rivulet becomes wide and shallow at the middle of the cylinder according to

$$
h_{\mathrm{m}} \sim \kappa_{N}\left(\alpha-\frac{\pi}{2}\right)^{\frac{N}{2(2 N+1)}} \rightarrow 0^{+}, \quad a \sim \pi\left(\alpha-\frac{\pi}{2}\right)^{-\frac{1}{2}} \rightarrow \infty
$$

in the limit $\alpha \rightarrow \pi / 2^{+}$, and that it becomes thick and of finite width near the bottom of the cylinder according to

$$
h_{\mathrm{m}} \sim \kappa_{N}(\pi-\alpha)^{-\frac{1}{2 N+1}} \rightarrow \infty, \quad a=\pi+\frac{\pi}{4}(\alpha-\pi)^{2}+O(\alpha-\pi)^{4} \rightarrow \pi^{+}
$$

in the limit $\alpha \rightarrow \pi^{-}$. Furthermore, $h_{\mathrm{m}} \sim 1 / \sin \alpha$ in the limit of a strongly shear-thinning fluid, $N \rightarrow 0^{+}$, and $h_{\mathrm{m}} \sim(8 / 3 \pi)^{1 / 2}(-\cos \alpha)^{1 / 4}$ in the limit of a strongly shear-thickening fluid, $N \rightarrow \infty$; 

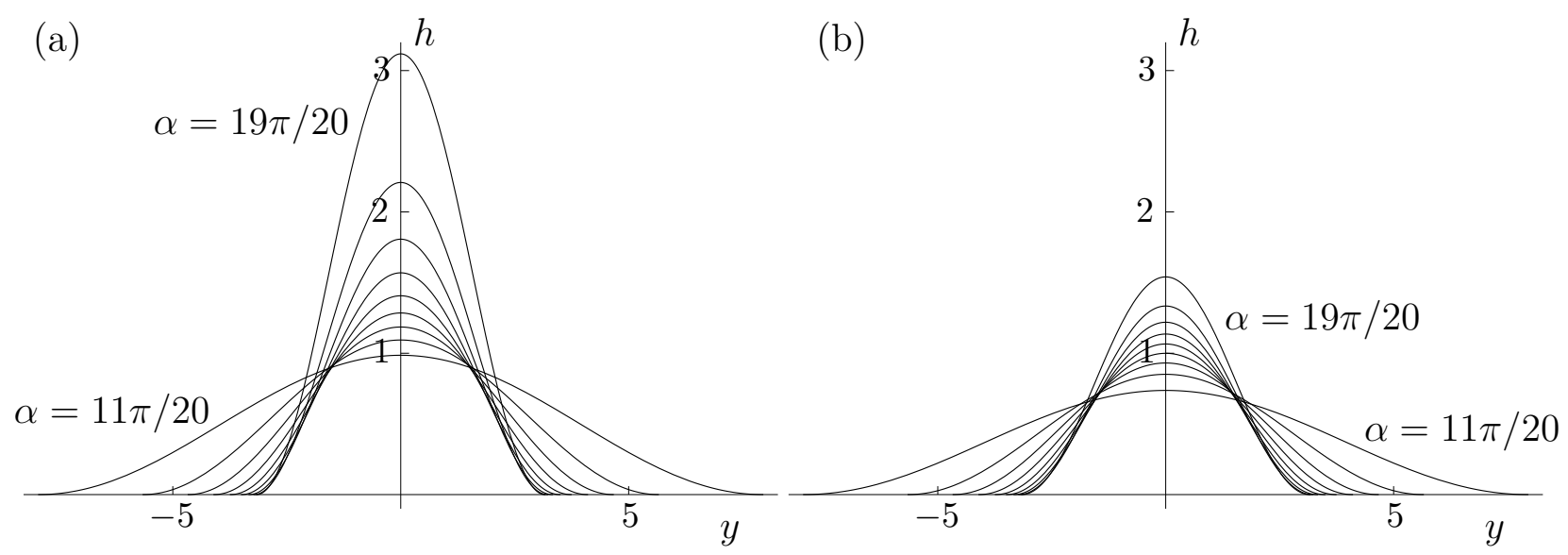

Figure 5: Plots of the cross-sectional free surface profile $h$ of a rivulet of a perfectly wetting fluid given by (9) as a function of $y$ at $\alpha=11 \pi / 20,12 \pi / 20, \ldots, 19 \pi / 20$ for (a) $N=1 / 2$ and (b) $N=2$.

these asymptotic solutions are also included in Figure 4 . Figure 4 also shows that the variation of $h_{\mathrm{m}}$ is not monotonic in $N$, in the sense that at any fixed value of $\alpha, h_{\mathrm{m}}$ increases with $N$ to a maximum value, and thereafter decreases monotonically with $N$.

Figure 5 shows plots of $h$ given by (9) as a function of $y$ at various stations around the cylinder for $N=1 / 2$ and $N=2$, and Figure 6 shows contour plots of $h$ in the $(y, \alpha / \pi)$ plane for the same values of $N$. In particular, Figures 5 and 6 illustrate that the forms of the rivulets for different values of $N$ are qualitatively similar.

Figure 7 shows contour plots of the velocity $u$ given by (3) when $\alpha=3 \pi / 4$ for $N=1 / 10$ and $N=10$, and shows that in the limits $N \rightarrow 0$ and $N \rightarrow \infty$ the velocity has the same qualitative behaviour as that described by Al Mukalal et al. [44] for a rivulet of a non-perfectly wetting fluid. In particular, Figure 7 (a) illustrates that in the limit $N \rightarrow 0$ we have

$$
u \sim N h(h \sin \alpha)^{\frac{1}{N}} \sim \frac{m \sin \alpha}{2 \sqrt{\pi N}}\left(\frac{1+\cos m y}{2}\right)^{\frac{1+N}{N}}
$$

except in a narrow boundary layer near the substrate, and hence that the flow "self-channels" down a narrow central channel defined by $h>1 / \sin \alpha$ with semi-width

$$
\frac{1}{m}\left(2 N \log \frac{m^{2} \sin ^{4} \alpha}{4 \pi N^{3}}\right)^{\frac{1}{2}}(\ll 1)
$$

between two "levées" of slowly moving fluid. In the particular case shown in Figure 7(a) the channel is defined by $h>\sqrt{2} \simeq 1.41421$ and has semi-width 0.86475. On the other hand, Figure 7(b) illustrates that in the limit $N \rightarrow \infty$ there is a simple linear velocity profile $u \rightarrow z$ except in a narrow boundary layer near the free surface. Figure 8 shows a plot of $u_{\max }$, namely

$$
u_{\max }=\frac{N}{N+1}(\sin \alpha)^{\frac{1}{2 N+1}} \kappa_{N}^{\frac{N+1}{N}} m^{\frac{N+1}{2 N+1}}
$$



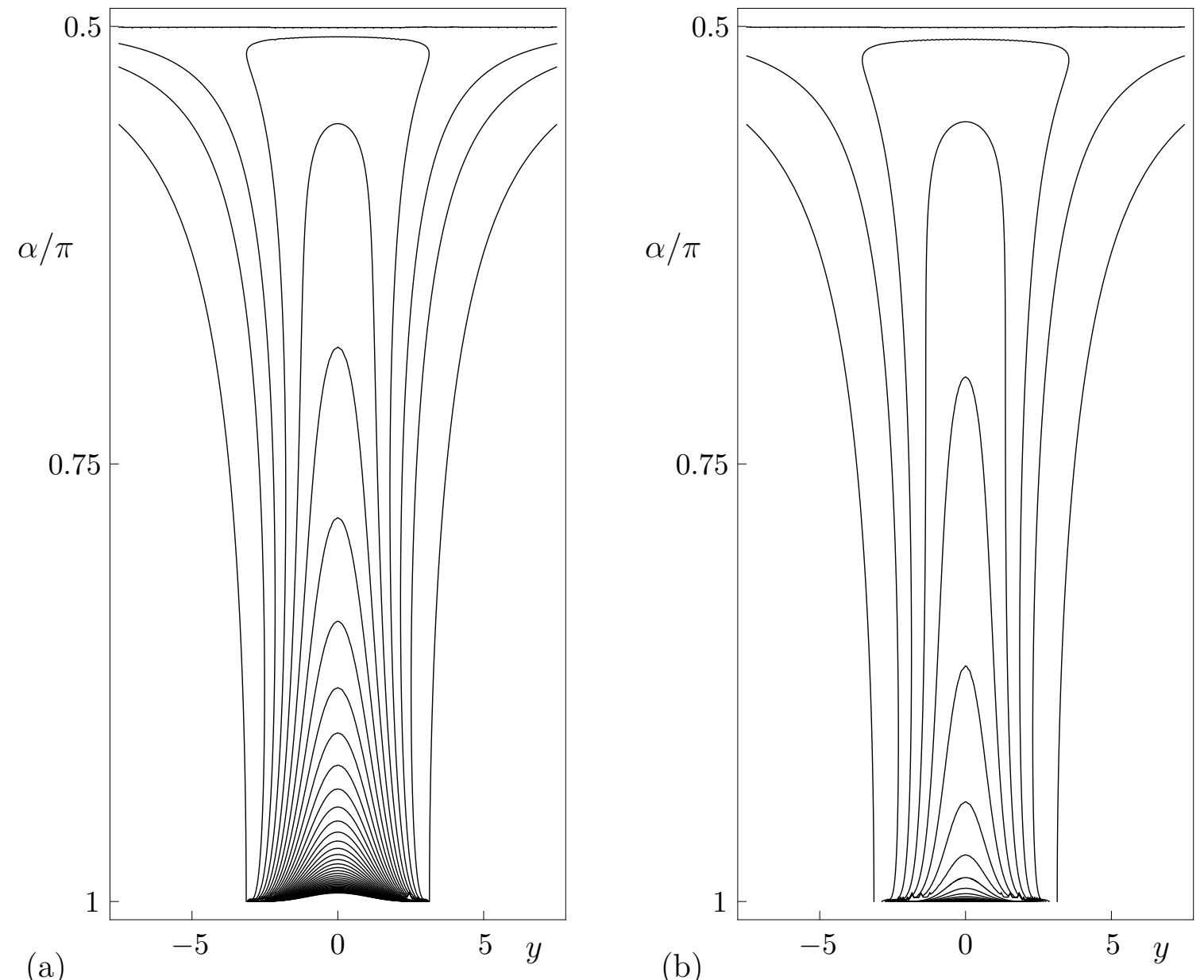

Figure 6: Contour plots of the free surface $h$ of a rivulet of a perfectly wetting fluid given by $(9)$ in the $(y, \alpha / \pi)$ plane for (a) $N=1 / 2$ and (b) $N=2$. The contours are drawn at intervals of $1 / 4$ up to a maximum value of $h=10$ in both cases.
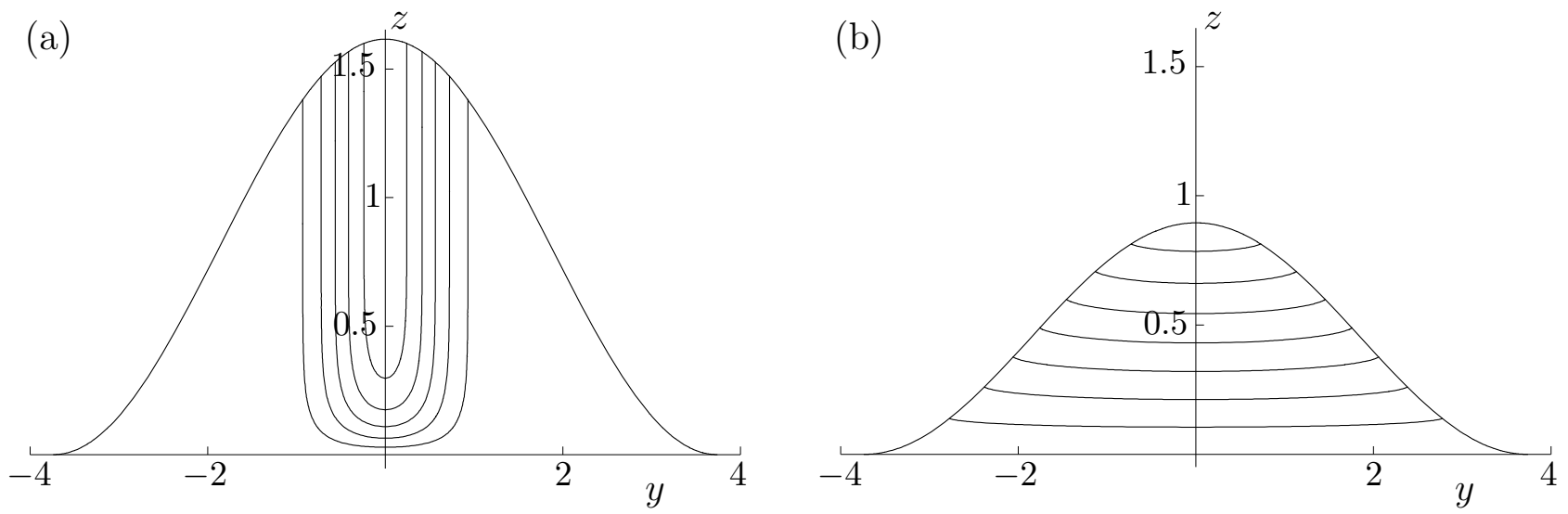

Figure 7: Contour plots of the velocity $u$ of a rivulet of a perfectly wetting fluid given by (3) when $\alpha=3 \pi / 4$ (in which case $a=\pi / m \simeq 3.73600$ ) for (a) $N=1 / 10$ (for which $h_{\mathrm{m}} \simeq 1.61671$ ) and (b) $N=10$ (for which $h_{\mathrm{m}} \simeq 0.89530$ ). The contour interval is 0.1 in both parts of the Figure. 


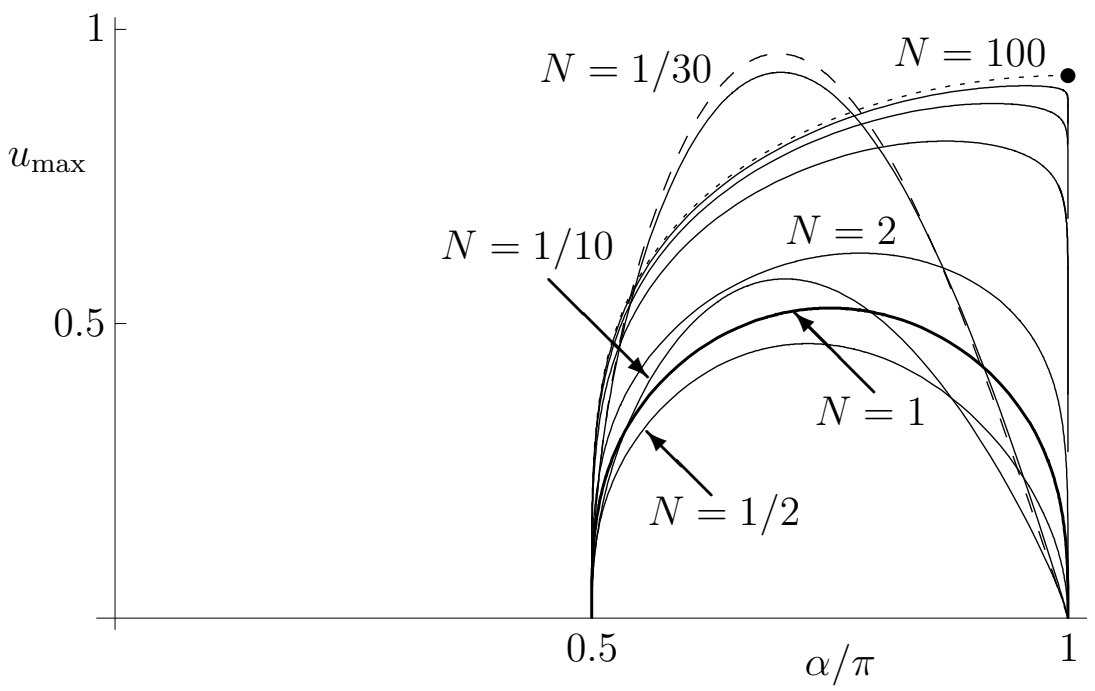

Figure 8: Plot of the maximum velocity $u_{\max }$ of a rivulet of a perfectly wetting fluid given by (20) as a function of $\alpha / \pi$ for $N=1 / 30,1 / 10,1 / 2,1$ (thicker curve), 2, 10, 30 and 100, together with the asymptotic solutions $u_{\max } \sim m \sin \alpha /(2 \sqrt{\pi N})$ in the limit $N \rightarrow 0$, drawn for the case $N=1 / 30$ (dashed curve), and $u_{\max }=(8 \mathrm{~m} / 3 \pi)^{1 / 2}$ in the limit $N \rightarrow \infty($ dotted curve).

as a function of $\alpha / \pi$ for a range of values of $N$, showing that $u_{\max }$ varies non-monotonically with both $\alpha$ and $N$. In particular, for any fixed value of $N, u_{\max }$ increases from zero at $\alpha=\pi / 2$ to a maximum value, and then decreases back to zero at $\alpha=\pi$, while at any fixed value of $\alpha, u_{\text {max }}$ decreases from $\infty$ (specifically, $u_{\max } \sim m \sin \alpha /(2 \sqrt{\pi N}) \rightarrow \infty$ as $N \rightarrow 0^{+}$) to a minimum value, and then increases to $(8 m / 3 \pi)^{1 / 2}$ as $N \rightarrow \infty$, taking the value $u_{\max }=2\left(9 m^{2} \sin \alpha /\left(25 \pi^{2}\right)\right)^{1 / 3}$ when $N=1$; these asymptotic solutions (the former drawn for the case $N=1 / 30$ ) are also included in Figure 8.

The mass of the rivulet, denoted by $M$ and non-dimensionalised by $\rho \epsilon \ell^{2} R$, where $R$ is the radius of the cylinder, is given by

$$
M=\int_{\pi / 2}^{\pi} \int_{-a}^{a} h \mathrm{~d} y \mathrm{~d} \alpha=\int_{\pi / 2}^{\pi} \frac{\pi h_{\mathrm{m}}}{m} \mathrm{~d} \alpha=\pi \kappa_{N} \int_{\pi / 2}^{\pi}\left((-\cos \alpha)^{\frac{N+1}{2}} \sin \alpha\right)^{-\frac{1}{2 N+1}} \mathrm{~d} \alpha
$$

leading to

$$
M=\frac{\pi}{2} \kappa_{N} \mathrm{~B}\left(\frac{N}{2 N+1}, \frac{3 N+1}{4(2 N+1)}\right) .
$$

Figure 9 shows a plot of $M$ as a function of $N$, illustrating that $M$ decreases monotonically with $N$, satisfying $M \sim \pi /(2 N) \rightarrow \infty$ in the limit $N \rightarrow 0^{+}, M=\left(3 \pi^{2} / 5\right)^{1 / 3} \mathrm{~B}(1 / 3,1 / 3) \simeq 9.58854$ when $N=1$ (in agreement with equations (9) and (10) of Paterson et al. [25]), and $M \rightarrow(2 \pi / 3)^{1 / 2} \mathrm{~B}(1 / 2,3 / 8)^{+} \simeq$ $5.58013^{+}$in the limit $N \rightarrow \infty$. 


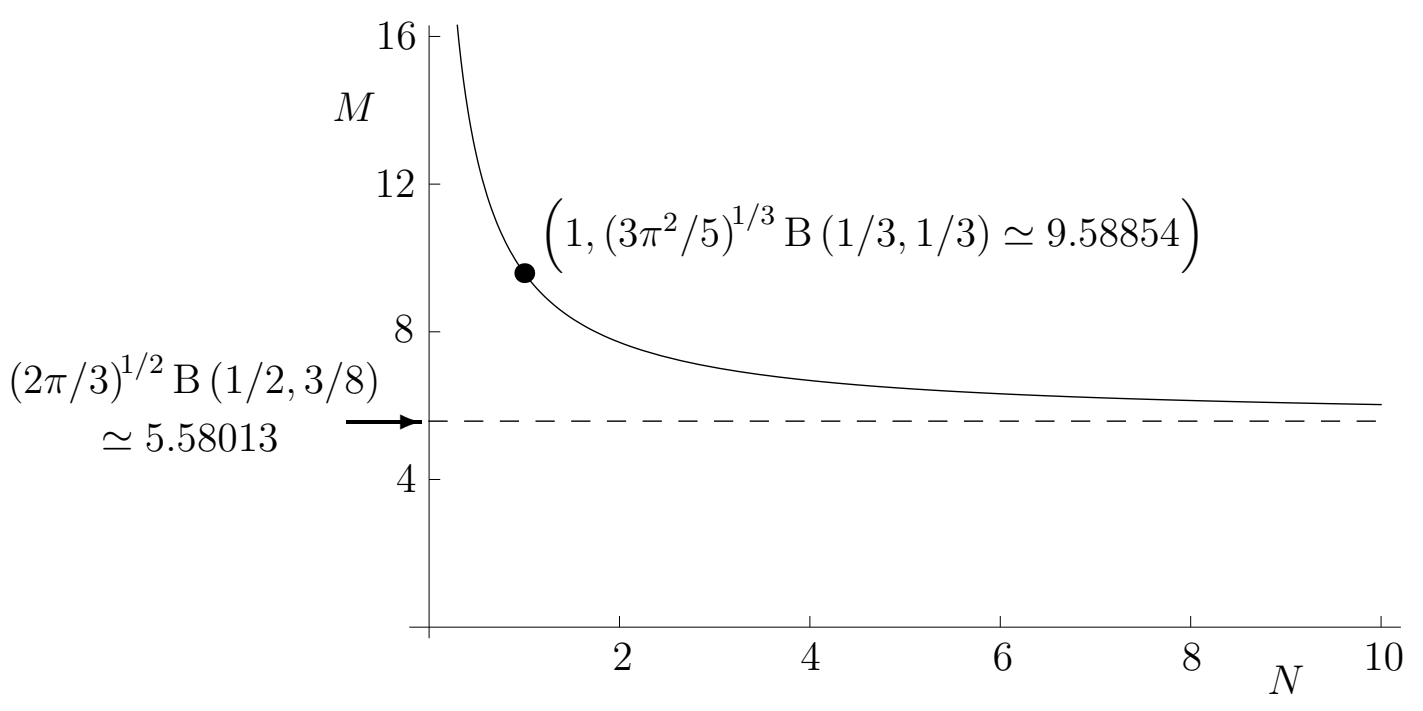

Figure 9: Plot of the mass of a rivulet of a perfectly wetting fluid, $M$, given by (22) as a function of $N$, together with its asymptotic value $(2 \pi / 3)^{1 / 2} \mathrm{~B}(1 / 2,3 / 8) \simeq 5.58013$ in the limit $N \rightarrow \infty$.

\section{A rivulet of a non-perfectly wetting fluid $(\beta>0)$}

In the general case of a non-perfectly wetting fluid $(\beta>0)$ the solution of $(5)$ and $(6)$ for $h$ is exactly the same as that for a Newtonian fluid (see, for example, Duffy and Moffatt [8]), namely

$$
h=\beta \times \begin{cases}\frac{\cosh m a-\cosh m y}{m \sinh m a} & \text { if } 0 \leq \alpha<\frac{\pi}{2}, \\ \frac{a^{2}-y^{2}}{2 a} & \text { if } \alpha=\frac{\pi}{2}, \\ \frac{\cos m y-\cos m a}{m \sin m a} & \text { if } \frac{\pi}{2}<\alpha \leq \pi .\end{cases}
$$

It may be shown that, whereas for $0 \leq \alpha \leq \pi / 2$ the solution (23) is valid for any $m a \geq 0$, for $\pi / 2<\alpha \leq \pi$ it is physically realisable only if $0 \leq m a<\pi$. The maximum thickness of the rivulet, $h_{\mathrm{m}}$, is obtained by setting $y=0$ in (23). As in the special case of a perfectly wetting fluid described in Sec. 4, although the form of the cross-sectional free surface profile of the rivulet is independent of $N$, its size (and, in particular, its contact angle $\beta$ ) depends on $N$ via the flux. Al Mukahal et al. [44] showed that the flux (7) is given by

$$
Q=\frac{1}{9}\left(\frac{\sin \alpha \beta^{2 N+1}}{m^{3 N+1}}\right)^{\frac{1}{N}} f_{N}(m a),
$$


where $f_{N}(m a)$ is defined by

$$
f_{N}(m a)=\lambda_{N} \times \begin{cases}{ }_{2} F_{1}\left(\frac{1}{2}, \frac{3 N+1}{N} ; \frac{7 N+2}{2 N} ; \tanh ^{2} \frac{m a}{2}\right) \tanh ^{\frac{3 N+1}{N}} \frac{m a}{2} & \text { if } 0 \leq \alpha<\frac{\pi}{2}, \\ \left(\frac{m a}{2}\right)^{\frac{3 N+1}{N}} & \text { if } \alpha=\frac{\pi}{2}, \\ { }_{2} F_{1}\left(\frac{1}{2}, \frac{3 N+1}{N} ; \frac{7 N+2}{2 N} ;-\tan ^{2} \frac{m a}{2}\right) \tan ^{\frac{3 N+1}{N}} \frac{m a}{2} & \text { if } \frac{\pi}{2}<\alpha \leq \pi,\end{cases}
$$

in which ${ }_{2} F_{1}$ denotes the hypergeometric function, and where $\lambda_{N}$ is defined by

$$
\lambda_{N}=\frac{18 \sqrt{\pi} \Gamma\left(\frac{2 N+1}{N}\right)}{\Gamma\left(\frac{7 N+2}{2 N}\right)}=36 \mathrm{~B}\left(\frac{3}{2}, \frac{2 N+1}{N}\right) .
$$

Al Mukahal et al. [44] plotted $\lambda_{N}$ as a function of $N$ and $f_{N}(m a)$ as a function of $m a$, and described their asymptotic behaviour.

Unlike Al Mukahal et al. [44], who prescribed the values of $Q$ and $\beta$ to obtain an implicit equation for the semi-width $a$, in the present work we prescribe the values of $Q$ and $a$ to determine the contact angle $\beta$. Specifically, setting $Q=\bar{Q}=1$ and $a=\bar{a}$ in (24) yields an explicit expression for $\beta$, namely

$$
\beta=\left(\frac{9^{N} m^{3 N+1}}{f_{N}(m \bar{a})^{N} \sin \alpha}\right)^{\frac{1}{2 N+1}},
$$

and $h$ is then given explicitly by (23). We note that $\beta$ (and hence the rivulet) does not have top-tobottom symmetry.

In the limit $N \rightarrow 0^{+}$we have

$$
\beta \sim \begin{cases}\frac{m}{\sin \alpha} \operatorname{coth} \frac{m \bar{a}}{2} & \text { if } 0 \leq \alpha<\frac{\pi}{2} \\ \frac{2}{\bar{a}} & \text { if } \alpha=\frac{\pi}{2} \\ \frac{m}{\sin \alpha} \cot \frac{m \bar{a}}{2} & \text { if } \frac{\pi}{2}<\alpha \leq \pi\end{cases}
$$

and $h_{\mathrm{m}}$ is then given by $h_{\mathrm{m}} \sim 1 / \sin \alpha$. On the other hand, in the limit $N \rightarrow \infty$ we have $f_{N}(m a) \rightarrow$ $f_{\infty}(m a)$, where

$$
f_{\infty}(m a)= \begin{cases}\frac{9}{2}\left(3 m a \operatorname{coth}^{2} m a-3 \operatorname{coth} m a-m a\right) & \text { if } 0 \leq \alpha<\frac{\pi}{2}, \\ \frac{6(m a)^{3}}{5} & \text { if } \alpha=\frac{\pi}{2}, \\ \frac{9}{2}\left(3 m a \cot ^{2} m a-3 \cot m a+m a\right) & \text { if } \frac{\pi}{2}<\alpha \leq \pi\end{cases}
$$

and hence (27) gives

$$
\beta \sim 3\left(\frac{m^{3}}{f_{\infty}(m \bar{a})}\right)^{\frac{1}{2}},
$$

and $h_{\mathrm{m}}$ is then given by setting $y=0$ in $(23)$. 


\subsection{A narrow rivulet with $\bar{a} \leq \pi$}

As we have already described in Sec. 3, Figure 1 shows a sketch of a narrow rivulet with prescribed constant semi-width $\bar{a} \leq \pi$. In particular, such a rivulet can flow all the way from the top to the bottom of the cylinder.

Figure 10 shows plots of $\beta$ and $h_{\mathrm{m}}$ for a narrow rivulet with prescribed constant semi-width $\bar{a}=2$ $(<\pi)$ as functions of $\alpha / \pi$ for a range of values of $N$. Figure 11 shows plots of $h$ given by (23) for a narrow rivulet with prescribed constant semi-width $\bar{a}=2(<\pi)$ as a function of $y$ at various stations around the cylinder for $N=1 / 2$. Figures 10 and 11 illustrate that the rivulet is thick at the top and bottom of the cylinder with both $\beta$ and $h_{\mathrm{m}}$ becoming infinite according to

$$
\beta \sim\left(\frac{9}{f_{N}(\bar{a})}\right)^{\frac{N}{2 N+1}} \alpha^{-\frac{1}{2 N+1}} \rightarrow \infty, \quad h_{\mathrm{m}} \sim\left(\frac{9}{f_{N}(\bar{a})}\right)^{\frac{N}{2 N+1}} \alpha^{-\frac{1}{2 N+1}} \tanh \frac{\bar{a}}{2} \rightarrow \infty
$$

in the limit $\alpha \rightarrow 0^{+}$, and

$$
\beta \sim\left(\frac{9}{f_{N}(\bar{a})}\right)^{\frac{N}{2 N+1}}(\pi-\alpha)^{-\frac{1}{2 N+1}} \rightarrow \infty, \quad h_{\mathrm{m}} \sim\left(\frac{9}{f_{N}(\bar{a})}\right)^{\frac{N}{2 N+1}}(\pi-\alpha)^{-\frac{1}{2 N+1}} \tan \frac{\bar{a}}{2} \rightarrow \infty
$$

in the limit $\alpha \rightarrow \pi^{-}$, except in the marginal case $\bar{a}=\pi$, in which $\beta=0$ at $\alpha=\pi$ and the rivulet becomes thick with zero contact angle and finite semi-width $\pi$ near the bottom of the cylinder according to

$$
\beta \sim \frac{\pi \kappa_{N}}{8}(\pi-\alpha)^{\frac{4 N+1}{2 N+1}} \rightarrow 0^{+}, \quad h_{\mathrm{m}} \sim \kappa_{N}(\pi-\alpha)^{-\frac{1}{2 N+1}} \rightarrow \infty
$$

in the limit $\alpha \rightarrow \pi^{-}$. Both $\beta$ and $h_{\mathrm{m}}$ are finite in the middle of the cylinder, satisfying

$$
\beta \rightarrow 2\left(\frac{18^{N}}{\lambda_{N}^{N} \bar{a}^{3 N+1}}\right)^{\frac{1}{2 N+1}}=O(1), \quad h_{\mathrm{m}} \rightarrow\left(\frac{18}{\lambda_{N} \bar{a}}\right)^{\frac{N}{2 N+1}}=O(1)
$$

in the limit $\alpha \rightarrow \pi / 2$. Stationary points of $\beta$ are determined mathematically by (24) and

$$
2 \csc ^{2} \alpha=N \frac{f_{N}^{\prime}(m a) m a}{f_{N}(m a)}-3 N+1
$$

(obtained by differentiation of (24) with respect to $\alpha$ ) from which it may be shown that $\beta$ has a unique minimum value, occurring on the lower half of the cylinder (i.e. for $\pi / 2<\alpha<\pi$ ). On the other hand, stationary points of $h_{\mathrm{m}}$ are determined mathematically by (24) and

$$
2 \csc ^{2} \alpha=N \frac{f_{N}^{\prime}(m a) m a}{f_{N}(m a)}-(2 N+1) \frac{2 m a}{\sinh 2 m a}+2-N
$$

(obtained by differentiation of $(23)$ with $y=0$ ) from which it may be shown that $h_{\mathrm{m}}$ also has a unique minimum value, but occurring on the upper half of the cylinder (i.e. for $0<\alpha<\pi / 2$ ). Furthermore, 

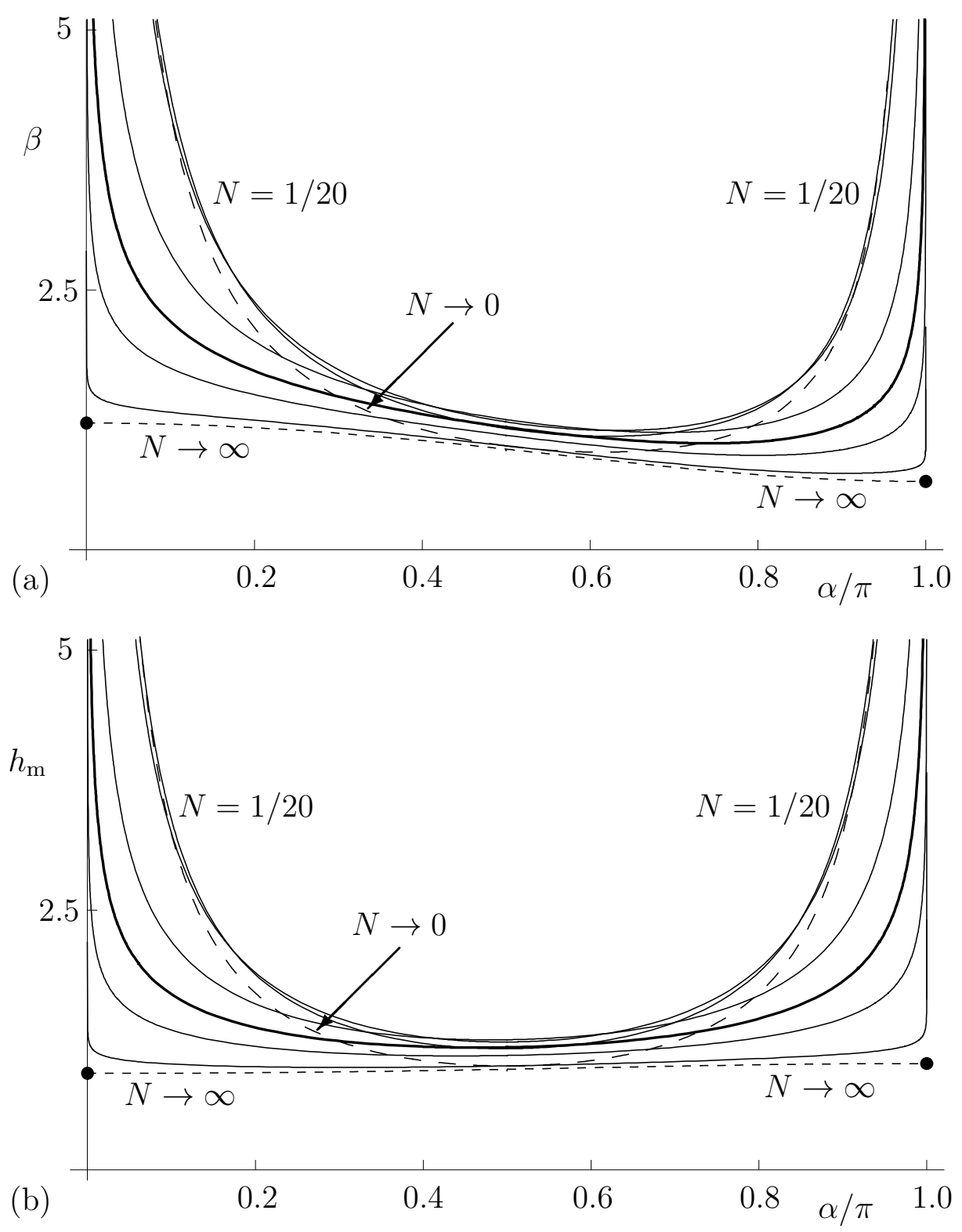

Figure 10: Plots of (a) the contact angle $\beta$ given by (27) and (b) the maximum thickness $h_{\mathrm{m}}$ given by setting $y=0$ in (23) of a narrow rivulet of a non-perfectly wetting fluid with prescribed constant semi-width $\bar{a}=2(<\pi)$ as functions of $\alpha / \pi$ for $N=1 / 20,1 / 10,1 / 2,1$ (thicker curve), 2 and 10, together with the asymptotic solutions (28) in the limit $N \rightarrow 0^{+}$(dashed curves), and (30) in the limit $N \rightarrow \infty$ (dotted curves). 
(a)

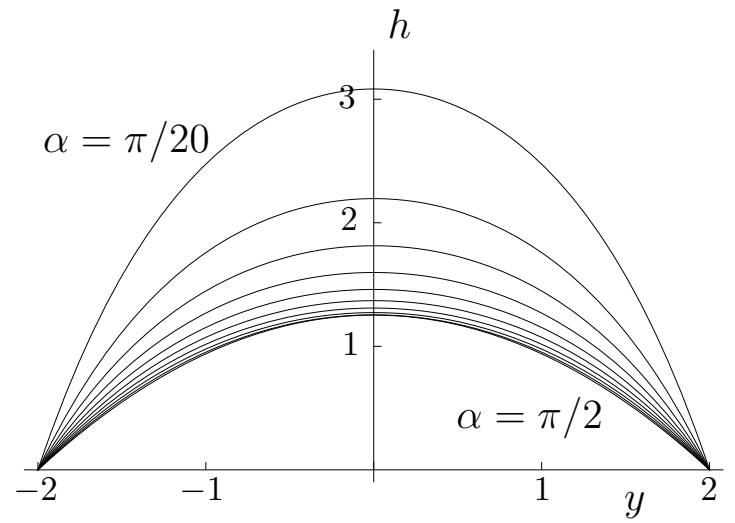

(b)

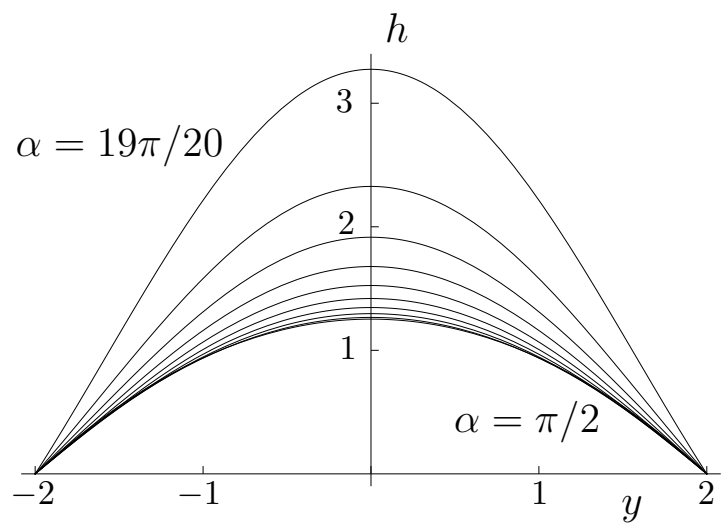

Figure 11: Plots of the cross-sectional free surface profile $h$ given by (23) for a narrow rivulet of a non-perfectly wetting fluid with prescribed constant semi-width $\bar{a}=2(<\pi)$ as a function of $y$ for $N=1 / 2$, at (a) $\alpha=\pi / 20, \pi / 10,3 \pi / 20, \ldots$, $\pi / 2$ on the top half of the cylinder, and (b) $\pi / 2,11 \pi / 20, \ldots, 19 \pi / 20$ on the bottom half of the cylinder.

Figure 10 also shows that, like the variation of $h_{\mathrm{m}}$ for a rivulet of a perfectly wetting fluid shown in Figure 4, the variation of $\beta$ and $h_{\mathrm{m}}$ is not monotonic in $N$ at any fixed value of $\alpha$.

In the limit of a very narrow rivulet, $\bar{a} \rightarrow 0^{+}$, the rivulet becomes thick according to

$$
\beta \sim 2\left(\frac{18^{N}}{\lambda_{N}^{N} \bar{a}^{3 N+1} \sin \alpha}\right)^{\frac{1}{2 N+1}} \rightarrow \infty, \quad h_{\mathrm{m}} \sim\left(\frac{18^{N}}{\lambda_{N}^{N} \bar{a}^{N} \sin \alpha}\right)^{\frac{1}{2 N+1}} \rightarrow \infty
$$

\subsection{A wide rivulet with $\bar{a}>\pi$}

As we have already described in Sec. 3, Figure 2 shows a sketch of a wide rivulet with prescribed constant semi-width $\bar{a}>\pi$. In particular, such a rivulet can flow all the way from the top to the bottom of the cylinder only if its contact lines de-pin at $\alpha=\alpha_{\text {depin }}\left(\pi / 2<\alpha_{\text {depin }}<\pi\right)$, where $\alpha_{\text {depin }}$ is given by (8), and thereafter it drains to the bottom of the cylinder with zero contact angle $\beta=0$ and slowly varying semi-width $a=\pi / m$ according to the solution for a rivulet of a perfectly wetting fluid described in Sec. 4.

Specifically, at $\alpha=\alpha_{\text {depin }}$ the rivulet has zero contact angle $\beta=0$, semi-width $a=\bar{a}$, and maximum thickness $h_{\mathrm{m}}=h_{\mathrm{mdepin}}$, where

$$
h_{\text {mdepin }}=\kappa_{N}\left(\frac{\left(-\cos \alpha_{\text {depin }}\right)^{\frac{N}{2}}}{\sin \alpha_{\text {depin }}}\right)^{\frac{1}{2 N+1}}=\kappa_{N}\left(\frac{\pi^{N} \bar{a}^{2-N}}{\sqrt{\bar{a}^{4}-\pi^{4}}}\right)^{\frac{1}{2 N+1}},
$$

while for $\alpha_{\text {depin }} \leq \alpha \leq \pi$ it has $\beta=0$, monotonically decreasing semi-width $a=\pi / m(\pi \leq a \leq \bar{a})$, and monotonically increasing maximum thickness $h_{\mathrm{m}}\left(\geq h_{\text {mdepin }}\right)$ given by (12).

In the limit $\alpha \rightarrow \alpha_{\text {depin }}^{-}$we have $a \equiv \bar{a}$, and find that $\beta \rightarrow 0^{+}$according to

$$
\beta=\left(\frac{\pi^{N}\left(\bar{a}^{4}-\pi^{4}\right)^{N}}{\bar{a}^{3 N-1}}\right)^{\frac{1}{2 N+1}} \frac{\kappa_{N}}{4}\left(\alpha_{\text {depin }}-\alpha\right)+O\left(\alpha_{\text {depin }}-\alpha\right)^{2}
$$


and $h_{\mathrm{m}} \rightarrow h_{\mathrm{mdepin}}^{-}$according to

$$
h_{\mathrm{m}}=h_{\mathrm{mdepin}}+\frac{\left(N \bar{a}^{4}+(2-N) \pi^{4}\right) h_{\text {mdepin }}}{2 \pi^{2} \sqrt{\bar{a}^{4}-\pi^{4}}}\left(\alpha-\alpha_{\text {depin }}\right)+O\left(\alpha_{\text {depin }}-\alpha\right)^{2},
$$

whereas in the limit $\alpha \rightarrow \alpha_{\text {depin }}^{+}$we have $\beta \equiv 0$, and find that $a \rightarrow \bar{a}^{-}$according to

$$
a=\bar{a}-\frac{\bar{a} \sqrt{\bar{a}^{4}-\pi^{4}}}{2 \pi^{2}}\left(\alpha-\alpha_{\text {depin }}\right)+O\left(\alpha_{\text {depin }}-\alpha\right)^{2}
$$

and $h_{\mathrm{m}} \rightarrow h_{\text {mdepin }}^{+}$according to (40). In particular, the solutions in $\alpha<\alpha_{\text {depin }}$ and $\alpha>\alpha_{\text {depin }}$ join continuously (but not smoothly) at $\alpha=\alpha_{\text {depin }}$.

Figure 12 shows plots of $a, \beta$ and $h_{\mathrm{m}}$ for a wide rivulet with prescribed constant semi-width $\bar{a}=5$ $(>\pi)$ in $0 \leq \alpha / \pi \leq \alpha_{\text {depin }} / \pi \simeq 0.62918$ and slowly varying semi-width $a=\pi / m$ in $\alpha_{\text {depin }} / \pi \leq \alpha / \pi \leq 1$ as functions of $\alpha / \pi$ for a range of values of $N$. Note that since $a$ takes the constant value $\bar{a}$ for all $\alpha$ when $\bar{a} \leq \pi$ and for $\alpha \leq \alpha_{\text {depin }}$ when $\bar{a}>\pi$, and is given by $a=\pi / m$ for $\alpha \geq \alpha_{\text {depin }}$ when $\bar{a}>\pi$, the plot of $a$ in Figure 12(a) is identical to the corresponding plot for Newtonian fluid given by Figure 6(b) of Paterson et al. [25], but is included here for completeness. Figure 13 shows plots of $h$ given by (9) and (23) for a wide rivulet with prescribed constant semi-width $\bar{a}=5(>\pi)$ as a function of $y$ at various stations around the cylinder, including $\alpha=\alpha_{\text {depin }} \simeq 0.62918 \pi$, for $N=1 / 2$. Figures 12 and 13 illustrate that the rivulet is again thick at the top of the cylinder with both $\beta$ and $h_{\mathrm{m}}$ becoming infinite according to $(31)$ in the limit $\alpha \rightarrow 0^{+}$, and they are again finite in the middle of the cylinder, satisfying (34) in the limit $\alpha \rightarrow \pi / 2$. However, in this case the rivulet becomes thick with zero contact angle and finite semi-width $\pi$ near the bottom of the cylinder according to (17) in the limit $\alpha \rightarrow \pi^{-}$. Futhermore, Figure 12 also shows that, like in the case of a narrow rivulet described in Sec. 5.1, the variation of $\beta$ and $h_{\mathrm{m}}$ is not monotonic in $N$ at any fixed value of $\alpha$.

In the limit of a very wide rivulet, $\bar{a} \rightarrow \infty$ (in which $\alpha_{\text {depin }} \rightarrow \pi / 2^{+}$), the rivulet becomes flat according to

$$
\beta \sim\left(\left(\frac{2 N+1}{2 N \bar{a}}\right)^{N} \frac{1}{\sin \alpha}\right)^{\frac{1}{2 N+1}} m \rightarrow 0^{+}, \quad h_{\mathrm{m}} \sim\left(\left(\frac{2 N+1}{2 N \bar{a}}\right)^{N} \frac{1}{\sin \alpha}\right)^{\frac{1}{2 N+1}} \rightarrow 0^{+}
$$

on the upper half of the cylinder and is given by (9) on the lower half of the cylinder.

\subsection{Contours plots of the free surface}

Figure 14 shows contour plots of $h$ given by (9) and (23) in the $(y, \alpha / \pi)$ plane for rivulets with prescribed semi-widths $\bar{a}=2$ and $\bar{a}=5$ when $N=1 / 2$. In particular, in the latter case the contact lines de-pin at $\alpha / \pi=\alpha_{\text {depin }} / \pi \simeq 0.62918$. 

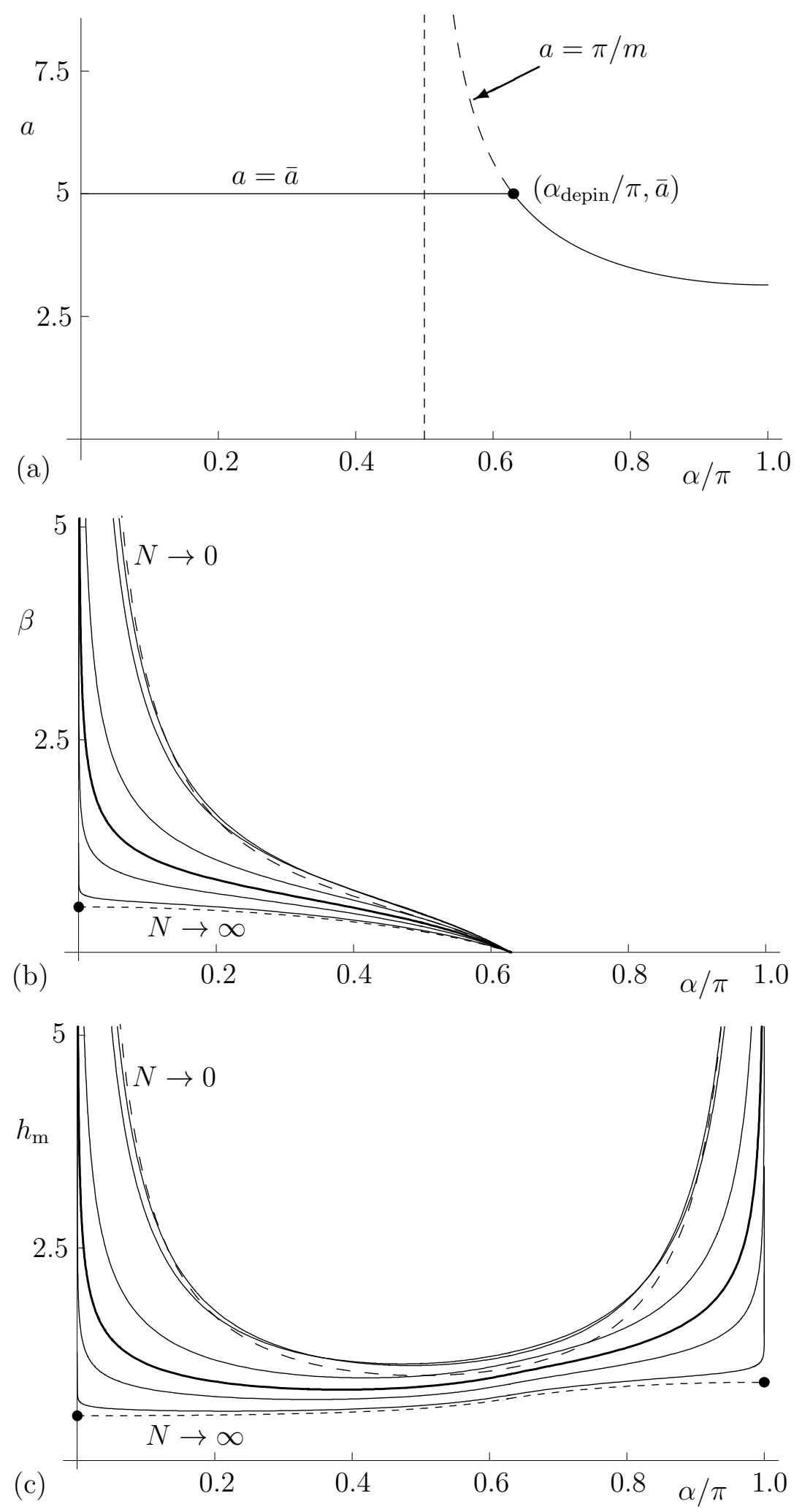

Figure 12: Plots of (a) $a$, (b) $\beta$ and (c) $h_{\mathrm{m}}$ for a wide rivulet of a non-perfectly wetting fluid with prescribed constant semi-width $\bar{a}=5(>\pi)$ in $0 \leq \alpha / \pi \leq \alpha_{\text {depin }} / \pi \simeq 0.62918$ and slowly varying semi-width $a=\pi / m$ in $\alpha_{\text {depin }} / \pi \leq \alpha / \pi \leq 1$ as functions of $\alpha / \pi$ for $N=1 / 20,1 / 10,1 / 2,1$ (thicker curve), 2 and 10, together with the asymptotic solutions (28) in the limit $N \rightarrow 0^{+}$(dashed curves), and (30) in the limit $N \rightarrow \infty$ (dotted curves). 

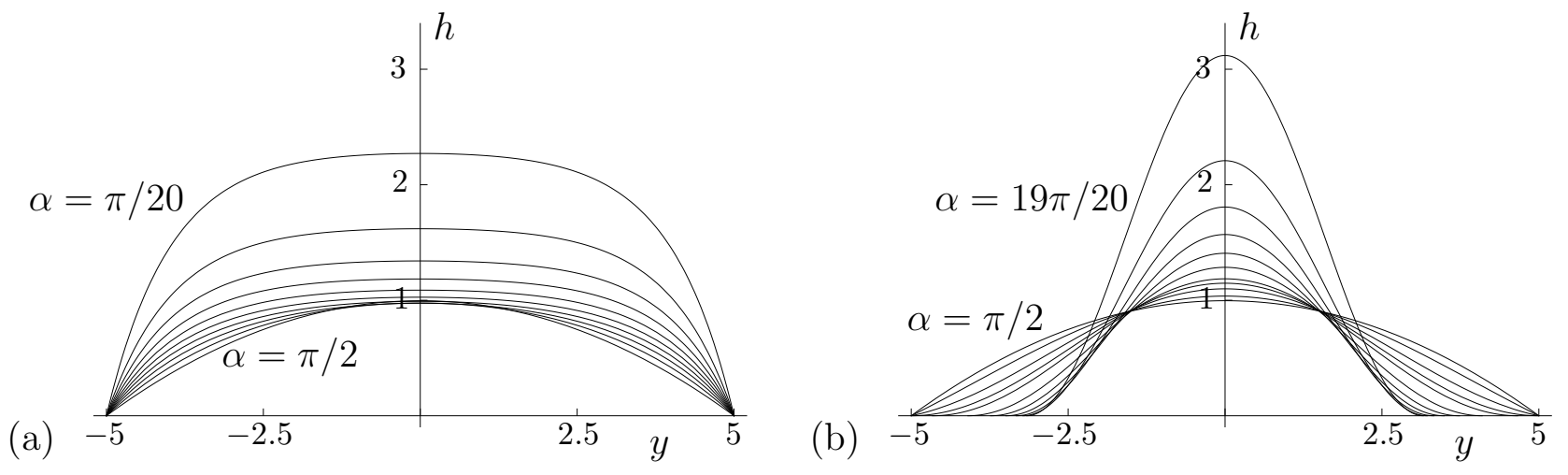

Figure 13: Plots of the cross-sectional free surface profile $h$ given by (9) and (23) for a wide rivulet of a non-perfectly wetting fluid with prescribed constant semi-width $\bar{a}=5(>\pi)$ as a function of $y$ for $N=1 / 2$ at (a) $\alpha=\pi / 20, \pi / 10$, $3 \pi / 20, \ldots, \pi / 2$, and (b) $\pi / 2,11 \pi / 20, \ldots, 19 \pi / 20$ and $\alpha=\alpha_{\text {depin }} \simeq 0.62918 \pi$.
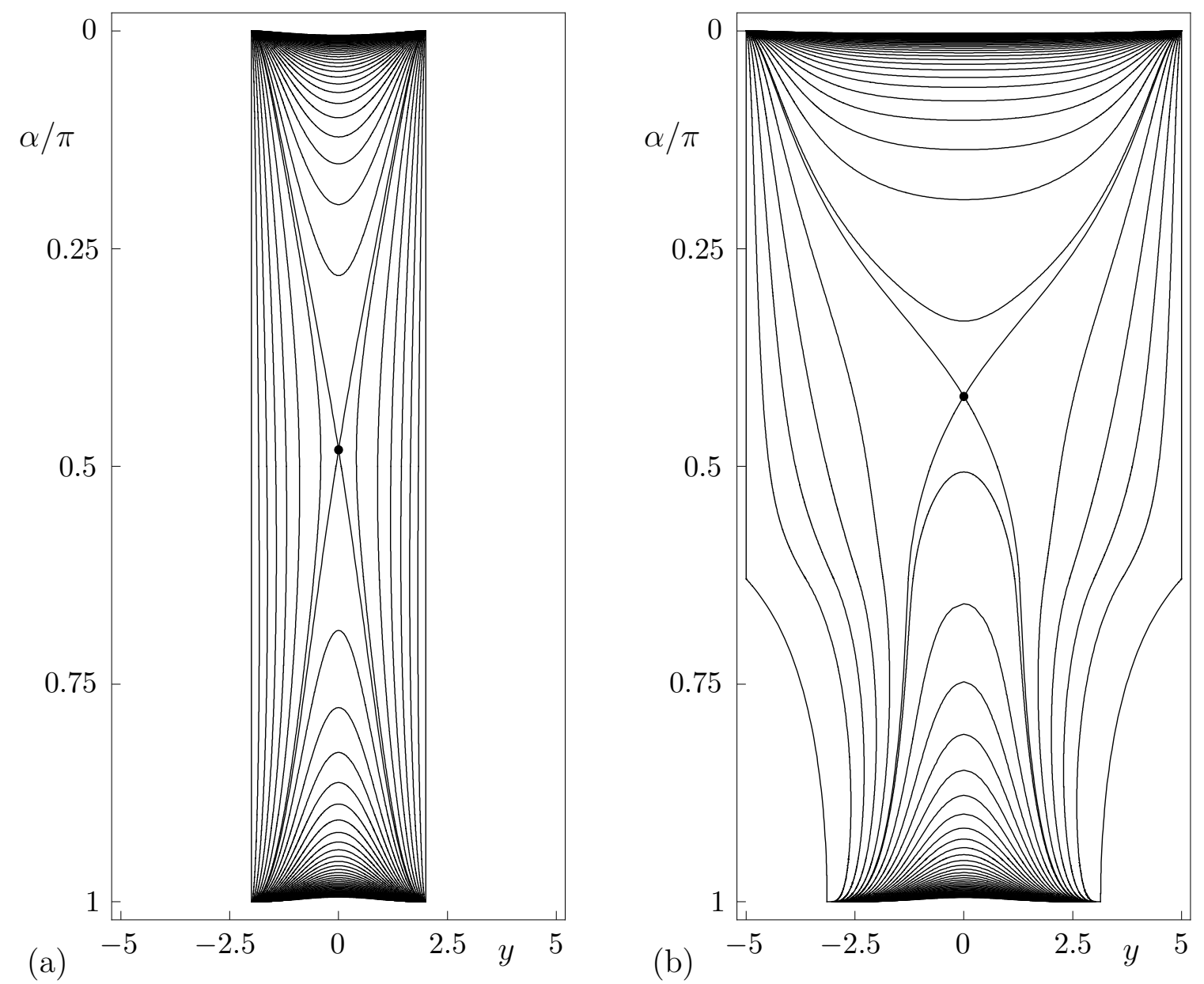

Figure 14: Contour plots of the free surface $h$ given by (9) and (23) in the $(y, \alpha / \pi)$ plane for rivulets of a non-perfectly wetting fluid with prescribed semi-widths (a) $\bar{a}=2$ and (b) $\bar{a}=5$ when $N=1 / 2$. In each case the contours are drawn at intervals of $1 / 5$ up to a maximum $h=10$, together with the contours (a) $h \simeq 1.25136$ and (b) $h \simeq 0.97273$ that pass through the saddle points of $h$, marked with dots. In (b) the contact lines de-pin at $\alpha / \pi=\alpha_{\text {depin }} / \pi \simeq 0.62918$. 


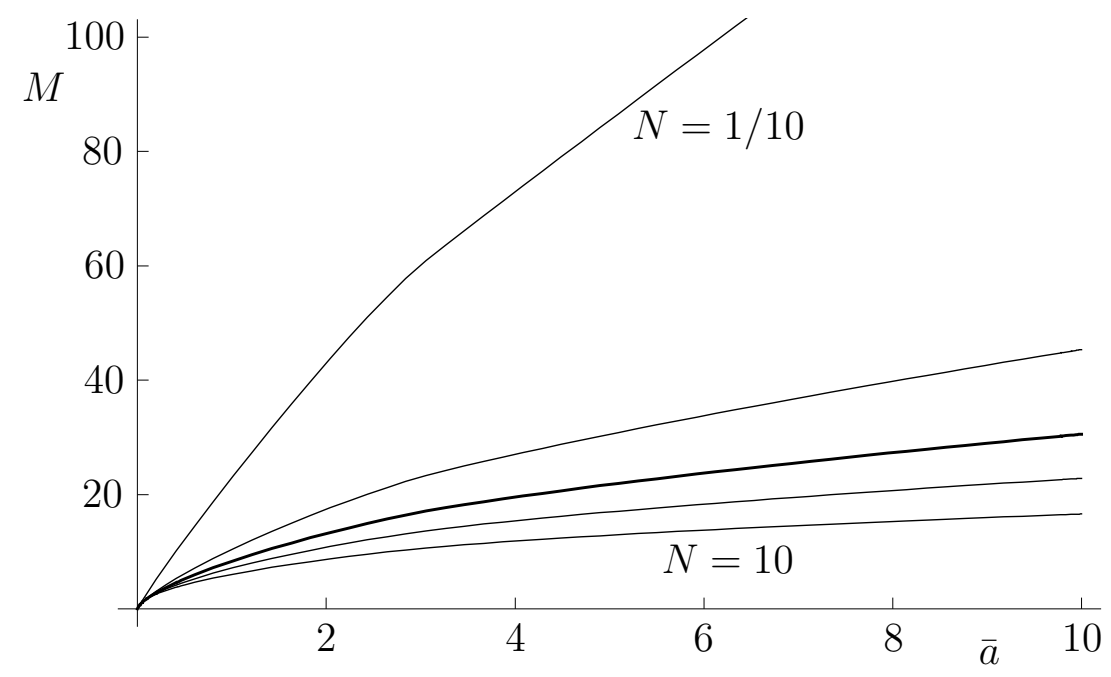

Figure 15: Plot of the mass of a rivulet of a non-perfectly wetting fluid, $M$, given by (43) as a function of $\bar{a}$ for $N=1 / 10,1 / 2,1$ (thicker curve), 2 and 10 .

\subsection{The mass of the rivulet}

Whatever the value of $\bar{a}$, the mass of the rivulet is given by

$$
M=\int_{0}^{\pi} \int_{-a}^{a} h \mathrm{~d} y \mathrm{~d} \alpha=\int_{0}^{\pi / 2} \frac{2 \beta(m a \operatorname{coth} m a-1)}{m^{2}} \mathrm{~d} \alpha+\int_{\pi / 2}^{\pi} \frac{2 \beta(1-m a \cot m a)}{m^{2}} \mathrm{~d} \alpha
$$

(although care must, of course, be exercised to evaluate the second integral correctly when $\bar{a}>\pi$ ). Figure 15 shows a plot of $M$ given by (43) as a function of $\bar{a}$ for several values of $N$, showing that $M$ increases monotonically with $\bar{a}$. In particular, in the limit of a narrow rivulet, $\bar{a} \rightarrow 0^{+}, M \rightarrow 0^{+}$ according to

$$
M \sim\left(\frac{2^{5 N+2} \bar{a}^{N+1}}{3 \lambda_{N}^{N}}\right)^{\frac{1}{2 N+1}} C_{N} \rightarrow 0^{+}
$$

whereas in the limit of a wide rivulet, $\bar{a} \rightarrow \infty, M \rightarrow \infty$ according to

$$
M \sim\left(\frac{(2 N+1)^{N} \bar{a}^{N+1}}{2^{N} N^{N}}\right)^{\frac{1}{2 N+1}} C_{N} \rightarrow \infty
$$

where the constant $C_{N}$ in both (44) and (45) is defined by

$$
C_{N}=\int_{0}^{\pi} \frac{\mathrm{d} \alpha}{(\sin \alpha)^{\frac{1}{2 N+1}}}=\frac{\sqrt{\pi} \Gamma\left(\frac{N}{2 N+1}\right)}{\Gamma\left(\frac{4 N+1}{2(2 N+1)}\right)}=\mathrm{B}\left(\frac{1}{2}, \frac{N}{2 N+1}\right) .
$$

Figure 16 shows a plot of $C_{N}$ as a function of $N$, illustrating that $C_{N}$ decreases monotonically with $N$, satisfying $C_{N} \sim 1 / N \rightarrow \infty$ as $N \rightarrow 0, C_{N}=\sqrt{\pi} \Gamma\left(\frac{1}{3}\right) / \Gamma\left(\frac{5}{6}\right) \simeq 4.20655$ when $N=1$ (in agreement with equation (10) of Paterson et al. [25]), and $C_{N} \rightarrow \pi^{+}$as $N \rightarrow \infty$. 


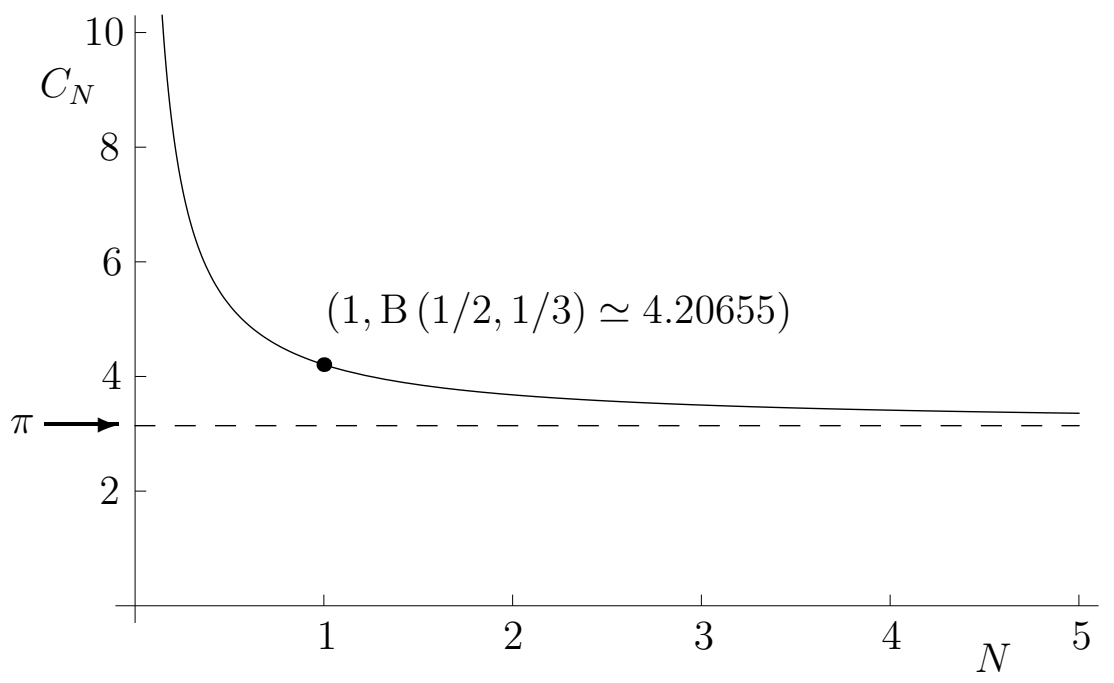

Figure 16: Plot of $C_{N}$ given by (46) as a function of $N$, together with its asymptotic value $\pi$ in the limit $N \rightarrow \infty$.

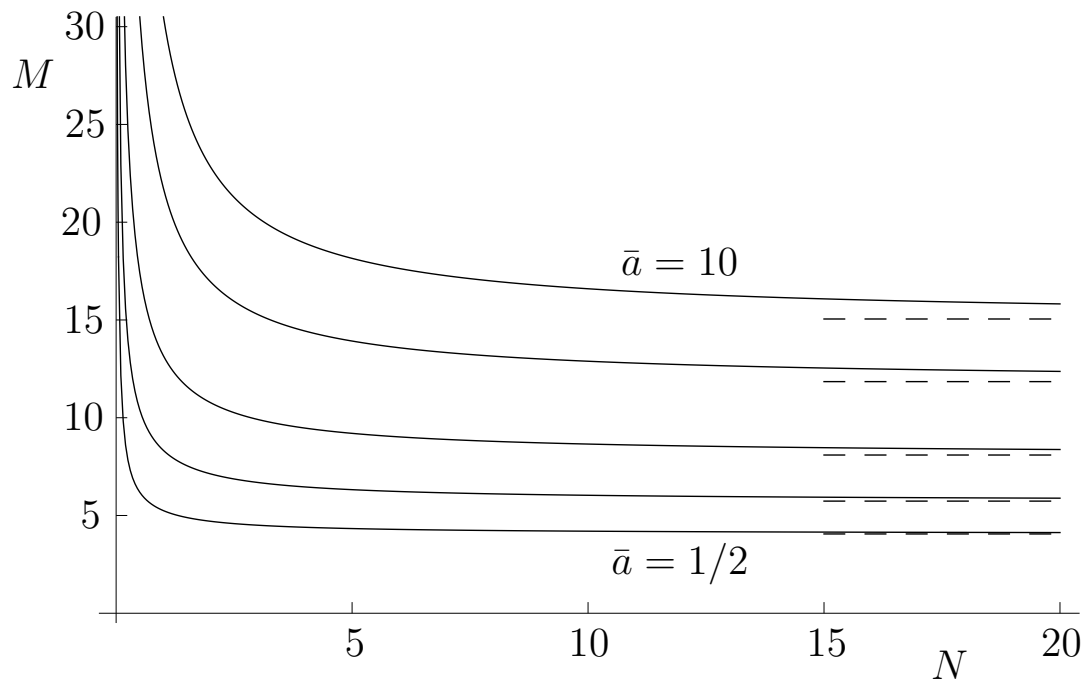

Figure 17: Plot of the mass of a rivulet of a non-perfectly wetting fluid, $M$, given by (43) as a function of $N$ for $\bar{a}=1 / 2$, 1, 2, 5 and 10. The horizontal dashed lines denote the constant asymptotic values in the limit $N \rightarrow \infty$. 
Figure 17 shows a plot of $M$ as a function of $N$ for several values of $\bar{a}$, showing that $M$ decreases monotonically with $N$ from $\infty$ to a (nonzero) $\bar{a}$-dependent constant asymptotic value in the limit $N \rightarrow \infty$.

\section{Conclusions}

In the present work we described the flow of a slowly varying rivulet of a power-law fluid with prescribed constant width (i.e. with pinned contact lines) but slowly varying contact angle down a slowly varying substrate, specifically the flow in the azimuthal direction around the outside of a large horizontal circular cylinder.

We obtained the solution for a rivulet of a perfectly wetting fluid (which can never have constant width), and showed that, despite having the same local behaviour, the global behaviour of a rivulet of a non-perfectly wetting fluid is qualitatively very different from that described by Al Mukahal et al. [44]. Specifically, we described how (as sketched in Figure 1) the contact lines of a sufficiently narrow rivulet with $\bar{a} \leq \pi$ can remain pinned as it drains all the way from the top to the bottom of the cylinder, but how (as sketched in Figure 2) the contact lines of a wider rivulet with $\bar{a}>\pi$ de-pin at $\alpha=\alpha_{\text {depin }}$ on the lower half of the cylinder, and how thereafter it drains to the bottom of the cylinder with zero contact angle $\beta=0$ and slowly varying semi-width $a=\pi / m$.

How the shape of the rivulet and the velocity within it depend on the power-law index $N$ was described in detail. In particular, we showed that whereas neither $h$ nor $u$ vary monotonically with $N$ at any fixed value of $\alpha$, its mass is always monotonically increasing in $\bar{a}$ but monotonically decreasing in $N$.

Despite the limitations of the power-law model, the present results provide rare analytical insight into non-Newtonian rivulet flow, and, in particular, are a useful benchmark for the study of rivulet flow of more realistic non-Newtonian fluids.

\section{Acknowledgements}

The first author (F.H.H.A.) wishes to thank the Ministry of Education, Kingdom of Saudi Arabia and King Faisal University for financial support via an Academic Staff Training Fellowship. The third author (S.K.W.) is supported by Leverhulme Trust Research Fellowship RF-2013-355 "Small Particles, Big Problems: Understanding the Complex Behaviour of Nanofluids' 
[1] N. Fraysse, G. M. Homsy, An experimental study of rivulet instabilities in centrifugal spin coating of viscous Newtonian and non-Newtonian fluids, Phys. Fluids 6 (1994) 1491-1504.

[2] N. J. Balmforth, R. V. Craster, R. Sassi, Shallow viscoplastic flow on an inclined plane, J. Fluid Mech. 470 (2002) 1-29.

[3] P. A. Nawrocki, K. T. Chuang, Carbon dioxide absorption into a stable liquid rivulet, Can. J. Chem. Eng. 74 (1996) 247-255.

[4] M. A. Herrada, A. S. Mohamed, J. M. Montanero, A. M. Gañán-Calvo, Stability of a rivulet flowing in a microchannel, Int. J. Multiphase Flow 69 (2015) 1-7.

[5] A. C. Robertson, I. J. Taylor, S. K. Wilson, B. R. Duffy, J. M. Sullivan, Numerical simulation of rivulet evolution on a horizontal cable subject to an external aerodynamic field, J. Fluids Structs 26 (2010) 50-73.

[6] G. D. Towell, L. B. Rothfeld, Hydrodynamics of rivulet flow, AIChE J. 12 (1966) 972-980.

[7] P. C. Smith, A similarity solution for slow viscous flow down an inclined plane, J. Fluid Mech. 58 (1973) 275-288.

[8] B. R. Duffy, H. K. Moffatt, Flow of a viscous trickle on a slowly varying incline, Chem. Eng. J. 60 (1995) 141-146.

[9] B. R. Duffy, H. K. Moffatt, A similarity solution for viscous source flow on a vertical plane, Eur. J. Appl. Math. 8 (1997) 37-47.

[10] D. Holland, B. R. Duffy, S. K. Wilson, Thermocapillary effects on a thin viscous rivulet draining steadily down a uniformly heated or cooled slowly varying substrate, J. Fluid Mech. 441 (2001) 195-221.

[11] B. R. Duffy, S. K. Wilson, A rivulet of perfectly wetting fluid with temperature-dependent viscosity draining down a uniformly heated or cooled slowly varying substrate, Phys. Fluids 15 (2003) 3236-3239.

[12] S. K. Wilson, B. R. Duffy, Strong temperature-dependent-viscosity effects on a rivulet draining down a uniformly heated or cooled slowly varying substrate, Phys. Fluids 15 (2003) 827-840.

[13] T. G. Myers, H. X. Liang, B. Wetton, The stability and flow of a rivulet driven by interfacial shear and gravity, Int. J. Nonlinear Mech. 39 (2004) 1239-1249. 
[14] S. K. Wilson, B. R. Duffy, A rivulet of perfectly wetting fluid draining steadily down a slowly varying substrate, IMA J. Appl. Math. 70 (2005) 293-322

[15] S. V. Alekseenko, A. V. Bobylev, D. M. Markovich, Rivulet flow on the outer surface of an inclined cylinder, J. Eng. Thermophys. 17 (2008) 259-272.

[16] E. S. Benilov, On the stability of shallow rivulets, J. Fluid Mech. 636 (2009) 455-474.

[17] J. A. Diez, A. G. González, L. Kondic, On the breakup of fluid rivulets, Phys. Fluids 21 (2009) 082105.

[18] J. A. Diez, A. G. González, L. Kondic, Instability of a transverse liquid rivulet on an inclined plane, Phys. Fluids 24 (2012) 032104.

[19] S. V. Alekseenko, A. V. Bobylev, V. V. Guzanov, D. M. Markovich, S. M. Kharlamov, Regular waves on vertical falling rivulets at different wetting contact angles, Thermophys. Aeromech. 17 (2010) 345-357.

[20] A. J. Tanasijczuk, C. A. Perazzo, J. Gratton, Navier-Stokes solutions for steady parallel-sided pendent rivulets, Euro. J. Mech. B / Fluids 29 (2010) 465-471.

[21] A. Daerr, J. Eggers, L. Limat, N. Valade, General mechanism for the meandering instability of rivulets of Newtonian fluids, Phys. Rev. Lett. 106 (2011) 184501.

[22] S. K. Wilson, J. M. Sullivan, B. R. Duffy, The energetics of the breakup of a sheet and of a rivulet on a vertical substrate in the presence of a uniform surface shear stress, J. Fluid Mech. 674 (2011) $281-306$.

[23] Y. M. Yatim, B. R. Duffy, S. K. Wilson, R. Hunt, Similarity solutions for unsteady gravity-driven slender rivulets, Q. J. Mech. Appl. Math. 64 (2011) 455-480.

[24] J. M. Sullivan, C. Paterson, S. K. Wilson, B. R. Duffy, A thin rivulet or ridge subject to a uniform transverse shear stress at its free surface due to an external airflow, Phys. Fluids 24 (2012) 082109.

[25] C. Paterson, S. K. Wilson, B. R. Duffy, Pinning, de-pinning and re-pinning of a slowly varying rivulet, Euro. J. Mech. B / Fluids 41 (2013) 94-108.

[26] C. Paterson, S. K. Wilson, B. R. Duffy, Rivulet flow round a horizontal cylinder subject to a uniform surface shear stress, Q. J. Mech. Appl. Math. 67 (2014) 567-597. 
[27] S. P. Aktershev, S. V. Alekseenko, Wave flow of a vertical rivulet, Tech. Phys. Lett. 41 (2015) $25-28$.

[28] S. V. Alekseenko, S. P. Aktershev, A. V. Bobylev, S. M. Kharlamov, D. M. Markovich, Nonlinear forced waves in a vertical rivulet flow, J. Fluid. Mech. 770 (2015) 350-373.

[29] S. Rosenblat, Rivulet flow of a viscoelastic liquid, J. Non-Newt. Fluid Mech. 13 (1983) 259-277.

[30] S. K. Wilson, B. R. Duffy, A. B. Ross, On the gravity-driven draining of a rivulet of viscoplastic material down a slowly varying substrate, Phys. Fluids 14 (2002) 555-571.

[31] S. D. R. Wilson, S. L. Burgess, The steady, spreading flow of a rivulet of mud, J. Non-Newt. Fluid Mech. 79 (1998) 77-85.

[32] S. K. Wilson, B. R. Duffy, R. Hunt, A slender rivulet of a power-law fluid driven by either gravity or a constant shear stress at the free surface, Q. J. Mech. Appl. Math. 55 (2002) 385-408.

[33] Y. M. Yatim, B. R. Duffy, S. K. Wilson, Unsteady gravity-driven slender rivulets of a power-law fluid, J. Non-Newt. Fluid Mech. 165 (2010) 1423-1430.

[34] R. W. Hewson, N. Kapur, P. H. Gaskell, A model for film-forming with Newtonian and shearthinning fluids, J. Non-Newt. Fluid Mech. 162 (2009) 21-28.

[35] A. J. Hogg, G. P. Matson, Slumps of viscoplastic fluids on slopes, J. Non-Newt. Fluid Mech. 158 (2009) 101-112.

[36] M. R. Chowdhury, F. Y. Testik, Viscous propagation of two-dimensional non-Newtonian gravity currents, Fluid. Dynam. Res. 44 (2012) 045502.

[37] A. Ganguly, M. Reza, A. S. Gupta, Thin-film flow of a power-law fluid down an inclined plane, J. Fluids Eng. 134 (2012) 044502.

[38] B. Hu, S. L. Kieweg, The effect of surface tension on the gravity-driven thin film flow of Newtonian and power-law fluids, Computers and Fluids 64 (2012) 83-90.

[39] J. Ahlkrona, N. Kirchner, P. Lötstedt, A numerical study of scaling relations for non-Newtonian thin-film flows with applications in ice sheet modelling, Q. J. Mech. Appl. Math. 66 (2013) 417435.

[40] V. O. Kheyfets, S. L. Kieweg, Gravity-driven thin film flow of an Ellis fluid, J. Non-Newt. Fluid Mech. 202 (2013) 88-98. 
[41] R. Sayag, M. G. Worster, Axisymmetric gravity currents of power-law fluids over a rigid horizontal surface, J. Fluid Mech. 716 (2013) R5.

[42] S. Fomin, R. Shankar, N. Danes, A. Yasuda, V. Chugunov, Rimming flow of a weakly elastic fluid, Theor. Comput. Fluid Dyn. 28 (2014) 485-498.

[43] D. Pritchard, B. R. Duffy, S. K. Wilson, Shallow flows of generalised Newtonian fluids on an inclined plane, to appear in J. Eng Math. (2015).

[44] F. H. H. Al Mukahal, B. R. Duffy, S. K. Wilson, A rivulet of a power-law fluid with constant contact angle draining down a slowly varying substrate, Phys. Fluids. 27 (2015) 052101. 